\title{
A phylogenetic framework for reassessing generic concepts and species delimitation in the lichenized family Trypetheliaceae (Ascomycota: Dothideomycetes)
}

\author{
Robert LÜCKING, Matthew P. NELSEN, André APTROOT, \\ Roselvira BARILLAS DE KLEE, Paulina A. BAWINGAN, Michel N. BENATTI, \\ Nguyen Quoc BINH, Frank BUNGARTZ, Marcela E. S. CACERES, \\ Luciana da Silva CANÊZ, José-Luis CHAVES, Damien ERTZ, \\ Rhina Esmeralda ESQUIVEL, Lidia Itati FERRARO, Alfredo GRIJALVA, \\ Cécile GUEIDAN, Jesús E. HERNÁNDEZ M., Allison KNIGHT, \\ H. Thorsten LUMBSCH, Marcelo P. MARCELLI, Joel A. MERCADO-DÍAZ, \\ Bibiana MONCADA, Eduardo A. MORALES, Khwanruan NAKSUWANKUL, \\ Thelma OROZCO, Sittiporn PARNMEN, Eimy RIVAS PLATA, \\ Noris SALAZAR-ALLEN, Adriano A. SPIELMANN and Nohemy VENTURA
}

\begin{abstract}
We provide an expanded and updated, 2-locus phylogeny (mtSSU, nuLSU) of the lichenized fungal family Trypetheliaceae, with a total of 196 ingroup OTUs, in order to further refine generic delimitations and species concepts in this family. As a result, the following 15 clades are recognized as separate genera, including five newly established genera: Aptrootia, Architrypethelium, Astrothelium (including the bulk of corticate species with astrothelioid ascospores; synonyms: Campylothelium, Cryptothelium, Laurera), Bathelium s. str. (excluding B. degenerans and relatives which fall into Astrothelium), the reinstated Bogoriella (for tropical, lichenized species previously placed in Mycomicrothelia), Constrictolumina gen. nov. (for tropical, lichenized species of Arthopyrenia), Dictyomeridium gen. nov. (for a subgroup of species with muriform ascospores previously placed in Polymeridium), fulella (provisionally, as the type species remains unsequenced), Marcelaria (Laurera purpurina complex), Nigrovothelium gen. nov. (for the Trypethelium tropicum group), Novomicrothelia gen. nov. (for an additional species previously placed in Mycomicrothelia),
\end{abstract}

R. Lücking: Botanical Garden and Botanical Museum Berlin, Königin-Luise-Straße 6-8, 14195 Berlin, Germany. Email: r.luecking@bgbm.org

R. Lücking, H. T. Lumbsch, J. A. Mercado-Díaz, M. P. Nelsen and E. Rivas Plata: Integrative Research Center, The Field Museum, 1400 South Lake Shore Drive, Chicago, Illinois 60605-2496, USA.

J. A. Mercado-Díaz: Committee on Evolutionary Biology, University of Chicago, 1025 E. 57th Street, Chicago, Illinois 60637, USA.

A. Aptroot: ABL Herbarium, G.v.d.Veenstraat 107, NL-3762 XK Soest, The Netherlands.

R. Barillas de Klee: Escuela de Biología, Facultad de Ciencias Químicas y Farmacia, Universidad de San Carlos de Guatemala, Zona 12, Guatemala City, Guatemala. P. A. Bawingan: School of Natural Sciences, Saint Louis University, Baguio City, Philippines.

M. N. Benatti and M. P. Marcelli: Instituto de Botânica, Núcleo de Pesquisa em Micologia, Caixa Postal 68041, 04045-972, São Paulo, Brazil.

N. Q. Binh: Vietnam Academy of Science and Technology, Vietnam National Museum of Nature, Department of Biology, 18 Hoang Quoc Viet Street, Cau Giay, Hanoi, Vietnam.
F. Bungartz: Fundación Charles Darwin, Puerto Ayora, Santa Cruz, Galápagos, Ecuador; mailing address: Schedestraße 8, 53113 Bonn, Germany.

M. E. S. Cáceres: Departamento de Biociências, Universidade Federal de Sergipe, CEP: 49.500-000, Itabaiana, Sergipe, Brazil.

L. S. Canêz and A. A. Spielmann: Laboratório de Liquenologia, Centro de Ciências Biológicas e da Saúde, Universidade Federal de Mato Grosso do Sul, Cidade Universitária, Caixa Postal 549, CEP 79070-900, Campo Grande, Mato Grosso do Sul, Brazil.

J.-L. Chaves: Laboratorio de Hongos, Instituto Nacional de Biodiversidad (INBio), Apdo. 22-3100, Santo Domingo de Heredia, Costa Rica.

D. Ertz: Department Bryophytes-Thallophytes (BT), Botanic Garden Meise, Nieuwelaan 38, B-1860 Meise, Belgium.

R. E. Esquivel and N. Ventura: Escuela de Biología, Facultad de Ciencias Naturales y Matemática, Universidad de El Salvador, Ciudad Universitaria, Final Av. Héroes y Mártires del 30 de Julio, San Salvador, El Salvador. 
Polymeridium s. str., Pseudopyrenula, Trypethelium s. str. (T. eluteriae group), and Viridothelium gen. nov. (for the Trypethelium virens group). All recognized genera are phenotypically characterized and a discussion on the evolution of phenotypic features in the family is given. Based on the obtained phylogeny, species delimitations are revised and the importance of characters such as thallus morphology, hymenial inspersion, and secondary chemistry for taxonomic purposes is discussed, resulting in a refined species concept.

Key words: evolution, genus concepts, lichenized fungi, phenotypic characters, species concepts

\section{Introduction}

Trypetheliaceae is one of the dominant elements of lichen communities in tropical rainforests, dry forests, and savannas (Komposch \& Hafellner 2000, 2003; Komposch et al. 2002; Aptroot et al. 2008, 2013; Aptroot $2009 a, b)$. The family was traditionally considered to belong in a suite of crustose, lichen-forming fungi with pyrenocarpous ascomata, and bitunicate (fissitunicate) asci, and was alternatively placed in the orders Pyrenulales or Melanommatales (Barr 1979, 1987; Harris 1984, 1991; Kirk et al. 2001; Eriksson et al. 2004; Cannon \& Kirk 2007). Eriksson (1981) elaborated a detailed scheme about the putative evolution of this group, postulating that a trypethelioid precursor, the so-called $\alpha$-Trypetheliaceae, gave rise to both the Pyrenulaceae and Trypetheliaceae. Pyrenulales are now classified near Verrucariales within the Eurotiomycetes, whereas Melanommatales have been synonymized with Pleosporales in the Dothideomycetes (Berbee 1996; Liew et al. 2000; Lumbsch \& Lindemuth 2001; Weerakoon et al. 2012), which generated ambiguity as to the correct placement of Trypetheliaceae. Inclusion of a single taxon in the first AFTOL study (Lutzoni et al. 2004) suggested placement of the family within Dothideomycetes, which was subsequently confirmed by Del Prado et al. (2006) with a more target-oriented taxon sampling, and by Nelsen et al. (2009) and Schoch et al. (2009) with phylogenetic studies focusing on Dothideomycetes. However, the family consistently clustered outside the Pleosporales and a separate order, Trypetheliales, was established for it (Aptroot et al. 2008). While Nelsen et al. (2009) demonstrated the close relationship between tropical Mycomicrothelia species and Trypetheliaceae,
L. I. Ferraro: Instituto de Botánica del Nordeste (IBONE), Casilla del Correo 209, 3400 Corrientes, Argentina.

A. Grijalva and T. Orozco: Herbario Nacional de Nicaragua, Universidad Centroamericana, Apartado 69, Managua, Nicaragua.

C. Gueidan: Department of Life Sciences, Natural History Museum, Cromwell Road, London, SW7 5BD, UK; and Australian National Herbarium, CSIRO, National Facilities and Collections, National Research Collections Australia, GPO Box 1600, Canberra, ACT 2601, Australia.

J. E. Hernández M.: Instituto Experimental Jardín Botánico Dr. Tobías Lasser, Universidad Central de Venezuela, Caracas, Venezuela.

A. Knight: Department of Botany, University of Otago, PO Box 56, Dunedin, New Zealand.
B. Moncada: Licenciatura en Biología, Universidad Distrital Francisco José de Caldas, Cra. 4 No. 26D-54, Torre de Laboratorios, Herbario, Bogotá, Colombia; Research Associate, Science and Education, The Field Museum, 1400 South Lake Shore, Chicago, IL 60605, USA.

E. A. Morales: Herbario Criptogámico Universidad Católica Boliviana, Carrera de Ingeniería Ambiental, Casilla de Correos 5381, Cochabamba, Bolivia.

K. Naksuwankul: Department of Biology and Natural Medicinal Mushroom Museum, Faculty of Science, Mahasarakham University, Kantarawichai, Maha Sarakham Province, 44150 Thailand.

S. Parnmen: Toxicology and Biochemistry Section, Department of Medical Sciences, Ministry of Public Health, Nonthaburi 11000, Thailand.

N. Salazar-Allen: Smithsonian Tropical Research Institute, Box 0843-03092, Balboa, Ancon, Panama. 
a subsequent phylogenetic study suggested inclusion of these species within the family, together with further species in the collective genera Arthopyrenia and fulella (Nelsen et al. 2011). A second family, Polycoccaceae, which chiefly includes lichenicolous fungi, has been recently established and included in Trypetheliales (Ertz et al. 2015).

Genera in Trypetheliaceae were traditionally separated by thallus structure, ascoma disposition, and ascospore type (Letrouit-Galinou 1957, 1958; Makhija \& Patwardhan 1988, 1993; Harris 1989a, b, 1990, 1991, 1995; Aptroot 1991a, b, 2009a, b; Aptroot et al. 2008; Lumbsch \& Huhndorf 2010). Thus, the core group with corticate thalli and typically astrothelioid ascospores (with diamond-shaped lumina) was divided into five genera according to ascoma disposition and ascospore septation: Trypethelium (solitary to aggregate ascomata with apical, separate ostioles, ascospores transversely septate), Laurera (solitary to aggregate ascomata with apical, separate ostioles, ascospores muriform), Astrothelium (solitary to aggregate ascomata with lateral, separate or fused ostioles, ascospores transversely septate), Cryptothelium (aggregate ascomata with lateral, fused ostioles, ascospores muriform), and Campylothelium (solitary ascomata with lateral, separate ostioles, ascospores muriform). In addition, the genus Pseudopyrenula was distinguished by its white, ecorticate thallus, combined with astrothelioid ascospores, whereas Polymeridium was defined as having an ecorticate thallus and thin-walled ascospores. This classification was not only considered artificial (Harris 1989a, 1995; Del Prado et al. 2006; Aptroot et al. 2008), but was also flawed logically, since species with separate or fused ostioles were united under a single genus (Astrothelium) if ascospores were transversely septate but separated into two genera (Campylothelium, Cryptothelium) if ascospores were muriform. Also, species in the Trypethelium eluteriae group have ascospores more similar to those of Polymeridium, lacking diamond-shaped lumina.

Harris $(1989 b, 1991,1995)$ was the first to try and establish more natural genera, such as Bathelium, characterized by a suite of morphoanatomical and chemical characters. The genera Aptrootia and Architrypethelium were introduced more recently for species with unique ascospore types (Aptroot 1991b; Lücking et al. 2007). Molecular phylogenetic studies then revealed the inclusion of some species of Arthopyrenia, Fulella, and Mycomicrothelia in a basal position in the family (Nelsen et al. 2009, 2011), all with morphologies similar to Polymeridium and Pseudopyrenula (with ecorticate thalli and mostly exposed ascomata).

Generic delimitation within Trypetheliaceae was evaluated in an expanded study (Nelsen et al. 2014), illustrating that a number of genera recognized within Trypetheliaceae were well defined, while the boundaries of several others required substantial adjustments. Thus, the bulk of the species with a corticate thallus and astrothelioid ascospores was suggested to be included in a single clade, Astrothelium, regardless of ascomatal disposition or ascospore septation; with such a modified concept, Cryptothelium and Laurera would become synonyms of Astrothelium, although no formal changes were proposed. In contrast, Trypethelium was to be retained for species in the $T$. eluteriae group, with aggregate, sessile pseudostromata and a unique ascospore type. The genera Aptrootia and Architrypethelium, and presumably also Campylothelium, were confirmed as monophyletic, whereas Bathelium s. str. with muriform ascospores formed a separate clade outside Astrothelium, with some species with transversely-septate ascospores previously assigned to this genus now included in Astrothelium. In addition, the genus Marcelaria was established for the enigmatic species Laurera purpurina and its relatives (Aptroot et al. 2013). Differences in ascospore type and ontogeny were found to reflect this modern understanding of phylogenetic relationships within the family (Sweetwood et al. 2012).

Species circumscriptions within Trypetheliaceae were also rather schematically based on ascospore size and septation, and the formation of anthraquinone and perylenequinone pigments, recognizing c. 200 taxa (Harris 1984; Aptroot 1991b; Del Prado et al. 2006; Aptroot et al. 2008). Characters such as hymenial inspersion, lichexanthone synthesis (Harris 
1991, 1995, 1998), and particularly thallus morphology were rarely, if at all, considered to be taxonomically important. The pigments, on the other hand, were subject to numerous rather detailed studies on their chemical structures and possible functions (Stensiö \& Wachtmeister 1969; Culberson \& Culberson 1970; Mathey \& Hoder 1978; Harris 1984; Mathey et al. 1987, 1994; Aptroot 1991b; Mathey \& Lukins 2001; Manojlovic et al. 2010).

Here we present a further, much expanded phylogenetic study based on two loci, the mitochondrial small subunit (mtSSU) and the nuclear large subunit (nuLSU) of the rDNA cistron, including a total of 196 operational taxonomic units (OTU's) spanning the entire diversity of the family. While providing a much-refined framework for revised generic delimitations, this study also focuses on the problem of species delimitation and the potential importance of phenotypic characters that have hitherto been neglected for taxonomic purposes in this family.

\section{Material and Methods}

We included all available mtSSU and nuLSU data on Trypetheliaceae published in previous studies (Lutzoni et al. 2004; Del Prado et al. 2006; Nelsen et al. 2009, 2011,2014 ) and added a total of 155 new sequences (100 mtSSU, 55 nuLSU) for 117 OTUs (Table 1). The only genera putatively placed within the family for which sequence data could not be obtained were Exiliseptum (Harris 1984) and Melanophloea (Aptroot \& Schumm 2012). Cladosporium cladosporioides was used as outgroup following Nelsen et al. (2014).

For newly generated sequences, the Sigma-Aldrich REDExtract-N-Amp Plant PCR Kit (St. Louis, Missouri, USA) was used to isolate DNA, following the manufacturer's instructions, except only $10-30 \mu \mathrm{l}$ of extraction buffer and 10-30 $\mu$ dilution buffer were used, and a $20 \times$ DNA dilution was then used in subsequent PCR reactions. A portion of the fungal mitochondrial small subunit ( $\mathrm{mtSSU}$ ) was amplified and sequenced using combinations of the following primers: mrSSU1, mrSSU2, mrSSU2R, mrSSU3R (Zoller et al. 1999), MSU7 (Zhou \& Stanosz 2001), mrSSU-1/2-5'-mpn and mrSSU-2/3-3'-mpn (Nelsen et al. 2011). Additionally, a portion of the fungal nuclear large subunit (nuLSU) was amplified and sequenced using combinations of the primers f-nu-LSU0116-5'/ITS4A-5' (Nelsen et al. 2011, 2012), AL2R (Mangold et al. 2008), f-nu-LSU-0287-5'-mpn (Nelsen et al. 2011), LR3 (Vilgalys \& Hester 1990), LR3R (reverse complement of LR3), LR4 (http://www.biology.duke.edu/ fungi/mycolab/primers.htm), LR5 and LR6 (Vilgalys \& Hester 1990).

The $10 \mu \mathrm{l}$ PCR reactions consisted of $5 \mu \mathrm{M}$ of each PCR primer, $3 \mathrm{mM}$ of each dNTP, $2 \mu \mathrm{l}$ of $10 \mathrm{mg} / \mathrm{ml} 100 \mathrm{X}$ BSA (New England BioLabs, Ipswich, Massachusetts, USA), $1.5 \mu 110 \times$ PCR buffer (Roche Applied Science, Indianapolis, Indiana, USA), $0.5 \mu \mathrm{l}$ Taq, approximately $2 \mu$ diluted DNA, and $2 \mu$ water or 2.5-5 $\mu$ l REDExtract-n-Amp PCR Ready Mix (Sigma-Aldrich, St. Louis, Missouri, USA), $5 \mu \mathrm{M}$ of each PCR primer, $2 \mu \mathrm{l}$ diluted DNA and $2-4.5 \mu \mathrm{l}$ water. The PCR cycling conditions were as follows: $95^{\circ} \mathrm{C}$ for $5 \mathrm{~min}$, followed by 35 cycles of $95^{\circ} \mathrm{C}$ for $1 \mathrm{~min}, 53^{\circ} \mathrm{C}$ (mtSSU), $55^{\circ} \mathrm{C}$ (nuLSU: AL2R/LR3) or $60^{\circ} \mathrm{C}$ (nuLSU: f-nu-LSU-0116-5'/ITS4A-5' with LR3 or LR6) for $1 \mathrm{~min}$, and $72{ }^{\circ} \mathrm{C}$ for $1 \mathrm{~min}$, followed by a single $72{ }^{\circ} \mathrm{C}$ final extension for $7 \mathrm{~min}$. Samples were visualized on an ethidium bromide-stained $1 \%$ agarose gel under UV light and bands were gel extracted, heated at $70{ }^{\circ} \mathrm{C}$ for $5 \mathrm{~min}$, cooled to $45{ }^{\circ} \mathrm{C}$ for $10 \mathrm{~min}$, treated with $1 \mu \mathrm{l}$ GELase (Epicentre Biotechnologies, Madison, Wisconsin, USA) and incubated at $45^{\circ} \mathrm{C}$ for at least $24 \mathrm{~h}$. The $10 \mu \mathrm{l}$ cycle sequencing reactions consisted of $1-1.5 \mu \mathrm{l}$ of Big Dye version 3.1 (Applied Biosystems, Foster City, California, USA), 2.5-3 $\mu \mathrm{l}$ of Big Dye buffer, $1-6 \mu \mathrm{M}$ primer (primers listed above), 0.75-2 $\mu$ l GELase-treated PCR product and water. Cycle sequencing was performed using one of the following conditions: $96^{\circ} \mathrm{C}$ for $1 \mathrm{~min}$, followed by 25 cycles of $96{ }^{\circ} \mathrm{C}$ for $10 \mathrm{~s}, 50{ }^{\circ} \mathrm{C}$ for $5 \mathrm{~s}$ and $60{ }^{\circ} \mathrm{C}$ for $4 \mathrm{~min}$ or instead $96^{\circ} \mathrm{C}$ for $1 \mathrm{~min}$, followed by 40 cycles of $96^{\circ} \mathrm{C}$ for $10 \mathrm{~s}, 45^{\circ} \mathrm{C}$ for $5 \mathrm{~s}$ and $60{ }^{\circ} \mathrm{C}$ for $4 \mathrm{~min}$. Samples were precipitated and sequenced in an Applied Biosystems 3730 DNA Analyzer (Foster City, California, USA), and sequences assembled in Sequencher 4.9 (Gene Codes Corporation, Ann Arbor, Michigan, USA). DNA analyses were performed at the Pritzker Laboratory for Molecular Systematics and Evolution at the Field Museum.

Sequences were automatically aligned using MAFFT 6.935 with sorting function (Katoh et al. 2009). The unaligned sequences were also subjected to assessment of alignment ambiguity through the Guidance web server (Penn et al. 2010a, b). No substantial ambiguity was detected for the nuLSU locus, whereas three large, ambiguously aligned regions were found in the $\mathrm{mtSSU}$ locus. These were removed from the alignment and separately recoded using PICS-Ord 1.0 (Lücking et al. 2011). After testing for potential conflict between the trimmed $\mathrm{mtSSU}$ and the nuLSU loci using Compat.py 3.0 (Kauff \& Lutzoni 2002, 2003), with no conflicts detected, the two loci were merged together with the PICS-Ord codes (see Supplementary Material A, available online) and a combined, partitioned maximum likelihood analysis (by locus and codes) was performed in RAxML 7.2.8 (Stamatakis 2006), employing a GTRGAMMA model for the nucleotide partitions and GTR for the code partition. Support was estimated by performing 1000 fast bootstrap pseudoreplicates (Stamatakis et al. 2008).

For selected portions of the resulting topology, we employed the Shimodeira-Hasegawa $(\mathrm{SH})$ test as implemented in RAxML 8.0.2 to test whether alternative topologies could be rejected. 
TABLE 1. GenBank Accession numbers and voucher information for taxa and samples used in this study. INB $\rightarrow$ CR indicates that the collections previously held at INB (National Biodiversity Institute, Costa Rica) are currently in the process of being transferred to CR (National Herbarium, Costa Rica).

\begin{tabular}{|c|c|c|c|c|c|c|c|}
\hline \multirow[b]{2}{*}{ Species } & \multirow[b]{2}{*}{ Extract } & \multirow[b]{2}{*}{ Country } & \multirow[b]{2}{*}{ Collector } & \multirow[b]{2}{*}{ Number } & \multirow[b]{2}{*}{ Repository } & \multicolumn{2}{|c|}{ GenBank Accession numbers } \\
\hline & & & & & & $\mathrm{mtSSU}$ & nuLSU \\
\hline Cladosporium cladosporioides & (outgroup) & & & & & FJ190628 & DQ678057 \\
\hline Aptrootia elatior & MPN560B & New Zealand & Knight & O61815 & (OTA) & KM453821 & KM4453754 \\
\hline A. robusta & MPN235B & Australia & Lumbsch & 20012 & (F) & KM453822 & KM453755 \\
\hline A. terricola & DNA1501 & Costa Rica & Lücking & 17211 & $(\mathrm{~F}, \mathrm{INB} \rightarrow \mathrm{CR})$ & DQ328995 & KM453756 \\
\hline Architrypethelium lauropaluanum & MPN48 & Peru & Nelsen & Cit1P & $(\mathrm{F})$ & KX215566 & KX215605 \\
\hline A. nitens & MPN257 & Panama & Lücking & 27038 & $(\mathrm{~F}, \mathrm{PMA})$ & KM453823 & KM453757 \\
\hline Astrothelium aeneum & MPN302 & Panama & Lücking & 27056 & $(\mathrm{~F}, \mathrm{PMA})$ & - & KX215606 \\
\hline A. aff. crassum & MPN335 & Brazil & Cáceres & 6011 & $(\mathrm{~F}, \mathrm{ISE})$ & KM453827 & KM453761 \\
\hline A. aff. megaspermum & MPN190 & Philippines & Rivas Plata & 2093 & (F, Saint Louis University) & KM453852 & KM453787 \\
\hline A. aff. norisianum & MPN23B & Peru & Nelsen & Cit1B & $(\mathrm{F})$ & KX215578 & KX215607 \\
\hline A. aff. obscurum & MPN194 & Philippines & Rivas Plata & 2175 & (F, Saint Louis University) & - & KX215608 \\
\hline A. aff. sepultum & MPN229 & Costa Rica & Lücking & 21027 & (F) & - & KX215609 \\
\hline$A$. aff. sepultum & MPN63C & Peru & Nelsen & $4001 \mathrm{a}$ & (F) & GU327690 & GU327714 \\
\hline A. bicolor & MPN139 & USA & Nelsen & $4002 \mathrm{a}$ & (F) & GU327706 & GU327728 \\
\hline A. carassense & MPN438 & Brazil & Lücking & 31004 & $(\mathrm{~F}, \mathrm{SP})$ & KM453849 & KM453784 \\
\hline A. cinereorosellum & MPN191 & Philippines & Rivas Plata & 2110 & (F, Saint Louis University) & KM453873 & KM453809 \\
\hline A. cinereorosellum & MPN199C & Philippines & Rivas Plata & 2106 & (F, Saint Louis University) & - & KX215610 \\
\hline A. cinnamomeum & AFTOL110 & Costa Rica & Lücking & $15322 b$ & (DUKE, F, INB $\rightarrow$ CR, USJ) & AY584632 & AY584652 \\
\hline A. crassum & MPN98 & Peru & Nelsen & s. n. & $(\mathrm{F})$ & GU327685 & GU327710 \\
\hline A. croceum & MPN55 & Peru & Nelsen & $211 \mathrm{D}$ & (F) & KX215567 & KX215611 \\
\hline A. degenerans & DNA1496 & Costa Rica & Lücking & $17502 b$ & $(\mathrm{CR}, \mathrm{F}, \mathrm{INB} \rightarrow \mathrm{CR}, \mathrm{USJ})$ & DQ328987 & - \\
\hline A. degenerans & DNA1497 & Costa Rica & Lücking & 16657 & (F) & DQ328988 & KX215612 \\
\hline A. degenerans & MPN266 & Panama & Lücking & $27131 x$ & (F, PMA) & - & KX215613 \\
\hline A. degenerans & MPN267 & Panama & Lücking & 27109 & $(\mathrm{~F}, \mathrm{PMA})$ & KM453835 & KM453770 \\
\hline A. degenerans & MPN397 & Galapagos & Rivas Plata & 4065 & $(\mathrm{CDS}, \mathrm{F})$ & KM453838 & KM453773 \\
\hline A. diplocarpum & MPN134 & USA & Nelsen & s. n. & $(\mathrm{F})$ & KX215568 & - \\
\hline A. diplocarpum & MPN210 & Nicaragua & Lücking & 28529 & $(\mathrm{~F}, \mathrm{HNMN})$ & KM453846 & KM453781 \\
\hline A. endochryseum & MPN436 & Brazil & Lücking & 31088 & $(\mathrm{~F}, \mathrm{SP})$ & KM453837 & KM453772 \\
\hline A. erubescens & MPN96 & Peru & Nelsen & AnaG & $(\mathrm{F})$ & KX215569 & KX215614 \\
\hline A. euthelium & MPN226 & Thailand & Lücking & 24075 & (F, RAMK) & - & KX215615 \\
\hline A. euthelium & MPN22B & Philippines & Rivas Plata & 1194B & $(\mathrm{F})$ & - & KX215616 \\
\hline A. floridanum & DNA1503 & Costa Rica & Lücking & $17090 \mathrm{~b}$ & $(\mathrm{~F}, \mathrm{USJ})$ & DQ329007 & - \\
\hline A. floridanum & MPN132 & USA & Nelsen & 4008 & $(\mathrm{~F})$ & GU327705 & GU327727 \\
\hline A. floridanum & MPN214 & Nicaragua & Lücking & 28558 & $(\mathrm{~F}, \mathrm{HNMN})$ & - & KX215617 \\
\hline
\end{tabular}




\begin{tabular}{|c|c|c|c|c|c|c|c|}
\hline \multirow[b]{2}{*}{ Species } & \multirow[b]{2}{*}{ Extract } & \multirow[b]{2}{*}{ Country } & \multirow[b]{2}{*}{ Collector } & \multirow[b]{2}{*}{ Number } & \multirow[b]{2}{*}{ Repository } & \multicolumn{2}{|c|}{ GenBank Accession numbers } \\
\hline & & & & & & $\mathrm{mtSSU}$ & nuLSU \\
\hline Astrothelium floridanum & MPN216 & Nicaragua & Lücking & 28564 & $(\mathrm{~F}, \mathrm{HNMN})$ & - & KX215618 \\
\hline A. floridanum & MPN304 & Panama & Lücking & $27131 \mathrm{a}$ & $(\mathrm{F}, \mathrm{PMA})$ & KM453876 & KM453811 \\
\hline A. gigantosporum & MPN590 & Panama & Lücking & 33037 & $(\mathrm{~F}, \mathrm{PMA})$ & KM453851 & KM453786 \\
\hline A. grossum & MPN259 & Panama & Lücking & 27045 & $(\mathrm{~F}, \mathrm{PMA})$ & KM453834 & KM453769 \\
\hline A. grossum & MPN262 & Panama & Lücking & 27083 & $(\mathrm{~F}, \mathrm{PMA})$ & - & KX215619 \\
\hline A. grossum & MPN263 & Panama & Lücking & $27131 v$ & (F, PMA) & - & KX215620 \\
\hline A. grossum & MPN264 & Panama & Lücking & 27084 & (F, PMA) & - & KX215621 \\
\hline A. grossum & MPN47 & Peru & Nelsen & $4000 \mathrm{a}$ & $(\mathrm{F})$ & GU327689 & GU327713 \\
\hline A. grossum & MPN657 & Panama & Nelsen & s. n. & $(\mathrm{F}, \mathrm{PMA})$ & KX215570 & KX215622 \\
\hline A. grossum & MPN703 & Brazil & Cáceres & 11137 & $(\mathrm{~F}, \mathrm{ISE})$ & - & KM453765 \\
\hline A. grossum & MPN751 & Puerto Rico & Mercado & 590 & $(\mathrm{~F}, \mathrm{UPR})$ & - & KX215623 \\
\hline A. grossum & MPN770 & Fiji & Lumbsch & $20556 h$ & $(\mathrm{~F})$ & KM453831 & KM453766 \\
\hline A. inspersoaeneum & MPN45 & Peru & Nelsen & Cit1K & $(\mathrm{F})$ & KX215571 & - \\
\hline A. kunzei & MPN201B & El Salvador & Lücking & 28120 & $(\mathrm{~F}, \mathrm{ITIC})$ & - & KX215624 \\
\hline A. kunzei & MPN203B & El Salvador & Lücking & 28137 & $(\mathrm{~F}, \mathrm{ITIC})$ & - & KX215625 \\
\hline A. laevigatum & MPN430 & Brazil & Lücking & 31010 & $(\mathrm{~F}, \mathrm{SP})$ & KX215572 & - \\
\hline A. laevithallinum & MPN442 & Brazil & Lücking & 31061 & $(\mathrm{~F}, \mathrm{SP})$ & KM453836 & KM453771 \\
\hline A. leucoconicum & MPN42 & Peru & Nelsen & $4000 c$ & $(\mathrm{~F})$ & KM453830 & KM453764 \\
\hline A. leucosessile & MPN258 & Panama & Lücking & 27059 & $(\mathrm{~F}, \mathrm{PMA})$ & KM453828 & KM453762 \\
\hline A. leucosessile & MPN265 & Panama & Lücking & 27093 & $(\mathrm{~F}, \mathrm{PMA})$ & - & KX215626 \\
\hline A. leucosessile & MPN58C & Peru & Nelsen & AnaE & $(\mathrm{F})$ & - & KX215627 \\
\hline A. leucosessile & MPN713 & Brazil & Cáceres & 11201 & $(\mathrm{~F}, \mathrm{ISE})$ & KM453869 & KM453805 \\
\hline A. macrocarpum & MPN131 & USA & Nelsen & s. n. & $(\mathrm{F})$ & KX215573 & KX215628 \\
\hline A. macrocarpum & MPN260 & Panama & Lücking & 27077 & $(\mathrm{~F}, \mathrm{PMA})$ & KM453829 & KM453763 \\
\hline A. macrocarpum & MPN261 & Panama & Lücking & 27052 & $(\mathrm{~F}, \mathrm{PMA})$ & - & KX215629 \\
\hline A. macrocarpum & MPN339 & Brazil & Cáceres & 6014 & $(\mathrm{~F}, \mathrm{ISE})$ & - & KX215630 \\
\hline A. macrocarpum & MPN57C & Peru & Nelsen & AnaA & $(\mathrm{F})$ & - & KX215631 \\
\hline A. megaspermum & AFTOL2094 & Gabon & Ertz & 9725 & (BR) & GU561847 & FJ267702 \\
\hline A. megaspermum & MPN138 & USA & Nelsen & s. n. & (F) & KX215574 & KX215632 \\
\hline A. megaspermum & MPN234 & Costa Rica & Lücking & s. n. & (F) & KX215575 & KX215633 \\
\hline A. megaspermum & MPN32B & Thailand & Nelsen & s. n. & $(\mathrm{F}, \mathrm{RAMK})$ & KX215576 & - \\
\hline A. meristosporum & MPN189 & Philippines & Rivas Plata & 2108 & ( $\mathrm{F}$, Saint Louis University) & KM453850 & KM453785 \\
\hline A. meristosporum & MPN198 & Philippines & Rivas Plata & 2128 & ( $\mathrm{F}$, Saint Louis University) & - & KX215634 \\
\hline A. neogalbineum & MPN51 & Peru & Nelsen & Cit1T & $(\mathrm{F})$ & KX215577 & KX215635 \\
\hline A. neogalbineum & MPN711 & Brazil & Cáceres & 11100 & $(\mathrm{~F}, \mathrm{ISE})$ & KM453877 & KM453812 \\
\hline A. neoinspersum & MPN61C & Peru & Nelsen & AnaJ & $(\mathrm{F})$ & - & KX215636 \\
\hline A. neoinspersum & MPN62 & Peru & Nelsen & s. n. & $(\mathrm{F})$ & KM453866 & KM453802 \\
\hline
\end{tabular}




\begin{tabular}{|c|c|c|c|c|c|c|c|}
\hline \multirow[b]{2}{*}{ Species } & \multirow[b]{2}{*}{ Extract } & \multirow[b]{2}{*}{ Country } & \multirow[b]{2}{*}{ Collector } & \multirow[b]{2}{*}{ Number } & \multirow[b]{2}{*}{ Repository } & \multicolumn{2}{|c|}{ GenBank Accession numbers } \\
\hline & & & & & & $\mathrm{mtSSU}$ & nuLSU \\
\hline Astrothelium nicaraguense & MPN205 & Nicaragua & Lücking & 28503 & $(\mathrm{~F}, \mathrm{HNMN})$ & - & KX215637 \\
\hline A. nicaraguense & MPN211 & Nicaragua & Lücking & 28546 & $(\mathrm{~F}, \mathrm{HNMN})$ & - & KX215638 \\
\hline A. nicaraguense & MPN213 & Nicaragua & Lücking & 28551 & $(\mathrm{~F}, \mathrm{HNMN})$ & - & KX215639 \\
\hline A. nitidiusculum & MPN704 & Brazil & Cáceres & 11297 & $(\mathrm{~F}, \mathrm{ISE})$ & KM453868 & KM453804 \\
\hline A. nitidiusculum & MPN768 & Fiji & Lumbsch & $20547 \mathrm{i}$ & $(\mathrm{F})$ & - & $\mathrm{KX} 215640$ \\
\hline A. norisianum & MPN52C & Peru & Nelsen & $4000 d$ & (F) & KM453848 & KM453783 \\
\hline A. obtectum & MPN422 & Brazil & Lücking & 31242 & $(\mathrm{~F}, \mathrm{SP})$ & KM453832 & KM453767 \\
\hline A. perspersum & AFTOL2099 & Gabon & Ertz & 9716 & (BR) & GU561848 & $\mathrm{FJ} 267701$ \\
\hline A. phlyctaena & MPN373 & USA & Nelsen & 4167 & $(\mathrm{~F})$ & - & KX215641 \\
\hline A. phlyctaena & MPN376 & USA & Nelsen & 4166 & (F) & - & $\mathrm{KX} 215642$ \\
\hline A. phlyctaena & MPN381 & USA & Nelsen & 4173 & (F) & - & $\mathrm{KX} 215643$ \\
\hline A. phlyctaena & MPN386 & USA & Nelsen & 4149 & (F) & - & KX215644 \\
\hline A. pulcherrimum & MPN313 & Panama & Lücking & 27046 & $(\mathrm{~F}, \mathrm{PMA})$ & KM453879 & KM453814 \\
\hline A. pupula & MPN224 & Colombia & Lücking & 26305 & (F, UDBC) & KM453880 & KM453815 \\
\hline A. purpurascens & MPN53C & Peru & Nelsen & s. n. & (F) & KM453847 & KM453782 \\
\hline A. robustum & MPN209 & Nicaragua & Lücking & 28519 & $(\mathrm{~F}, \mathrm{HNMN})$ & - & KX215645 \\
\hline A. robustum & MPN212 & Nicaragua & Lücking & 28547 & $(\mathrm{~F}, \mathrm{HNMN})$ & - & KX215646 \\
\hline A. robustum & MPN221 & Guatemala & Lücking & 25558 & $(\mathrm{BIGU}, \mathrm{F})$ & - & KX215647 \\
\hline A. robustum & MPN222 & Guatemala & Lücking & 25559 & $(\mathrm{BIGU}, \mathrm{F})$ & - & KX215648 \\
\hline A. robustum & MPN223 & Guatemala & Lücking & 25534 & (BIGU, F) & - & KX215649 \\
\hline A. robustum & MPN754 & Costa Rica & Mercado & 586 & (F) & KM453826 & KM453760 \\
\hline A. rufescens & MPN143 & Brazil & Nelsen & $\mathrm{B} 1$ & $(\mathrm{~F}, \mathrm{SP})$ & - & KX215650 \\
\hline A. rufescens & MPN345 & Argentina & Lücking & 30510 & (CTES, F) & - & KX215651 \\
\hline A. rufescens & MPN346 & Argentina & Lücking & 30511 & (CTES, F) & - & KX215652 \\
\hline A. sanguinarium & MPN765 & Brazil & Cañez & 3133 & (CGMS, F) & KM453853 & KM453788 \\
\hline A. sanguinarium & MPN766 & Brazil & Cañez & 3135 & (CGMS, F) & KX215579 & KX215653 \\
\hline A. sanguinarium & MPN767 & Brazil & Cañez & $3137 \mathrm{a}$ & (CGMS, F) & KX215580 & KX215654 \\
\hline A. scoria & MPN310 & Panama & Lücking & 27181 & $(\mathrm{~F}, \mathrm{PMA})$ & - & KX215655 \\
\hline A. scorizum & MPN336 & Brazil & Lücking & 29814 & $(\mathrm{~F}, \mathrm{SP})$ & KM453872 & KM453808 \\
\hline Astrothelium sp. (pycnidia) & MPN133 & USA & Nelsen & s. n. & $(\mathrm{F})$ & KX215581 & KX215656 \\
\hline Astrothelium sp. (pycnidia) & MPN162 & Brazil & Nelsen & $\mathrm{B} 20$ & $(\mathrm{~F}, \mathrm{SP})$ & KX215582 & $\mathrm{KX} 215657$ \\
\hline Astrothelium sp. (pycnidia) & MPN390 & USA & Nelsen & $4082 \mathrm{~A}$ & $(\mathrm{~F})$ & - & $\mathrm{KX} 215658$ \\
\hline A. subcatervarium & MPN97 & Peru & Nelsen & $4009 a$ & (F) & GU327707 & GU327729 \\
\hline A. subendochryseum & MPN202B & El Salvador & Lücking & 28121 & (F, ITIC) & - & KX215659 \\
\hline A. subinterjectum & MPN157 & Brazil & Nelsen & $\mathrm{B} 15$ & $(\mathrm{~F}, \mathrm{SP})$ & KX215583 & $\mathrm{KX} 215660$ \\
\hline A. subscoria & MPN217 & Nicaragua & Lücking & 28640 & $(\mathrm{~F}, \mathrm{HNMN})$ & KM453878 & KM453813 \\
\hline A. subscoria & MPN325 & Bolivia & Lücking & 29010 & (F) & KX215584 & KX215661 \\
\hline
\end{tabular}




\begin{tabular}{|c|c|c|c|c|c|c|c|}
\hline \multirow[b]{2}{*}{ Species } & \multirow[b]{2}{*}{ Extract } & \multirow[b]{2}{*}{ Country } & \multirow[b]{2}{*}{ Collector } & \multirow[b]{2}{*}{ Number } & \multirow[b]{2}{*}{ Repository } & \multicolumn{2}{|c|}{ GenBank Accession numbers } \\
\hline & & & & & & $\mathrm{mtSSU}$ & nuLSU \\
\hline Astrothelium tuberculosum & DNA1504 & Costa Rica & Lücking & $16306 a$ & $(\mathrm{~F}, \mathrm{INB} \rightarrow \mathrm{CR})$ & DQ329008 & - \\
\hline A. variolosum & MPN41 & Peru & Nelsen & Cit $1 F$ & $(\mathrm{~F})$ & KX215585 & KX215662 \\
\hline A. variolosum & MPN43 & Peru & Nelsen & s. n. & (F) & KM453833 & KM453768 \\
\hline Bathelium lineare & MPN741 & Vietnam & Gueidan & 2078 & (F) & KM453839 & KM453774 \\
\hline B. madreporiforme & MPN354 & Brazil & Lücking & 23290 & $(\mathrm{~F}, \mathrm{SP})$ & KM453840 & KM453775 \\
\hline B. madreporiforme & MPN355 & Brazil & Lücking & 23271 & $(\mathrm{~F}, \mathrm{SP})$ & - & KX215663 \\
\hline B. madreporiforme & MPN356 & Brazil & Lücking & 23248 & $(\mathrm{~F}, \mathrm{SP})$ & - & KX215664 \\
\hline B. porinosporum & MPN743 & Vietnam & Gueidan & 3040 & $(\mathrm{~F})$ & KM453841 & KM453776 \\
\hline B. porinosporum & MPN744 & Vietnam & Gueidan & 3055 & (F) & KX215586 & KX215665 \\
\hline B. porinosporum & MPN745 & Vietnam & Gueidan & 3041 & (F) & - & KX215666 \\
\hline B. porinosporum & MPN747 & Vietnam & Gueidan & 3053 & (F) & KX215587 & KX215667 \\
\hline B. tuberculosum & MPN112 & India & Lumbsch & $19733 \mathrm{j}$ & $(\mathrm{F})$ & - & KX215668 \\
\hline B. tuberculosum & MPN113 & India & Lumbsch & $19739 z$ & $(\mathrm{~F})$ & - & KX215669 \\
\hline B. tuberculosum & MPN81 & India & Lumbsch & $19739 z$ & (F) & KM453842 & KM453777 \\
\hline Bogoriella hemisphaerica & MPN102 & Nicaragua & Lücking & 28641 & $(\mathrm{~F}, \mathrm{HNMN})$ & GU327695 & GU327719 \\
\hline B. miculiformis & MPN101B & Nicaragua & Lücking & 28637 & $(\mathrm{~F}, \mathrm{HNMN})$ & GU327696 & GU327720 \\
\hline B. minutula & MPN567 & Thailand & Nelsen & s. n. & $(\mathrm{F}, \mathrm{RAMK})$ & KM453856 & - \\
\hline Constrictolumina bifera & MPN574 & Thailand & Nelsen & s. n. & $(\mathrm{F}, \mathrm{RAMK})$ & KM453824 & - \\
\hline C. cinchonae & MPN333 & Brazil & Lücking & 29583 & $(\mathrm{~F}, \mathrm{SP})$ & JN872349 & JN872351 \\
\hline C. cinchonae & MPN417 & Brazil & Lücking & s. n. & $(\mathrm{F}, \mathrm{SP})$ & KM453825 & KM453759 \\
\hline C. planorbis & MPN330 & Brazil & Lücking & 29532 & $(\mathrm{~F}, \mathrm{SP})$ & - & KX215670 \\
\hline C. planorbis & MPN331 & Brazil & Lücking & 29587 & $(\mathrm{~F}, \mathrm{SP})$ & - & KX215671 \\
\hline C. planorbis & MPN332 & Brazil & Lücking & 29585 & $(\mathrm{~F}, \mathrm{SP})$ & - & KX215672 \\
\hline C. planorbis & MPN334 & Brazil & Lücking & 29584 & $(\mathrm{~F}, \mathrm{SP})$ & JN872350 & JN872352 \\
\hline C. planorbis & MPN352 & Brazil & Lücking & 23289 & $(\mathrm{~F}, \mathrm{SP})$ & - & JN872353 \\
\hline C. planorbis & MPN353 & Brazil & Lücking & 23228 & $(\mathrm{~F}, \mathrm{SP})$ & - & $\mathrm{JN} 872354$ \\
\hline Constrictolumina sp. & MPN575 & Thailand & Nelsen & s. n. & $(\mathrm{F}, \mathrm{RAMK})$ & KX215588 & - \\
\hline Dictyomeridium proponens & MPN359 & Venezuela & Lücking & 26103 & $(\mathrm{~F}, \mathrm{VEN})$ & KM453860 & JN887403 \\
\hline Fulella fallaciosa & MPN141 & USA & Nelsen & s. n. & $(\mathrm{F})$ & JN887411 & JN887399 \\
\hline 7. fallaciosa & MPN547 & USA & Nelsen & s. n. & (F) & JN887412 & JN887400 \\
\hline Marcelaria cumingii & MPN552 & Thailand & Parnmen & s. n. & (F) & KM453854 & KM453789 \\
\hline M. purpurina & MPN323A & Brazil & Cáceres & 2009 & $(\mathrm{~F}, \mathrm{ISE})$ & KM453855 & KM453790 \\
\hline Nigrovothelium bullatum & MPN114 & India & Lumbsch & $19701 \mathrm{~g}$ & (F) & KX215589 & KX215673 \\
\hline N. bullatum & MPN579 & Thailand & Parnmen & 32 & (F) & KX215590 & KX215674 \\
\hline N. bullatum & MPN82 & India & Lumbsch & $19701 \mathrm{~g}$ & (F) & KX215591 & KX215675 \\
\hline N. tropicum & MPN130 & USA & Nelsen & s. n. & (F) & KM453883 & KM453819 \\
\hline N. tropicum & MPN195 & Philippines & Rivas Plata & 2155 & (F, Saint Louis University) & - & KX215676 \\
\hline
\end{tabular}




\begin{tabular}{|c|c|c|c|c|c|c|c|}
\hline \multirow[b]{2}{*}{ Species } & \multirow[b]{2}{*}{ Extract } & \multirow[b]{2}{*}{ Country } & \multirow[b]{2}{*}{ Collector } & \multirow[b]{2}{*}{ Number } & \multirow[b]{2}{*}{ Repository } & \multicolumn{2}{|c|}{ GenBank Accession numbers } \\
\hline & & & & & & $\mathrm{mtSSU}$ & nuLSU \\
\hline Nigrovothelium tropicum & MPN197 & Philippines & Rivas Plata & 2173 & (F, Saint Louis University) & - & KX215677 \\
\hline N. tropicum & $\mathrm{MPN} 25$ & Thailand & Nelsen & 4003 & $(\mathrm{~F}, \mathrm{RAMK})$ & GU327708 & GU327730 \\
\hline N. tropicum & MPN318 & Panama & Lücking & 27092 & $(\mathrm{~F}, \mathrm{PMA})$ & - & KX215678 \\
\hline N. tropicum & MPN44 & Peru & Nelsen & Cit1J & (F) & KX215592 & KX215679 \\
\hline N. tropicum & MPN561 & Thailand & Nelsen & s. n. & (F, RAMK) & KX215593 & KX215680 \\
\hline N. tropicum & MPN658 & Panama & Nelsen & s. n. & $(\mathrm{F}, \mathrm{PMA})$ & KX215594 & - \\
\hline Novomicrothelia oleosa & MPN700 & Brazil & Cáceres & 11821 & $(\mathrm{~F}, \mathrm{ISE})$ & KM453857 & KM453794 \\
\hline N. oleosa & MPN95 & Peru & Nelsen & $4007 \mathrm{a}$ & $(\mathrm{F})$ & GU327697 & GU327721 \\
\hline Polymeridium albocinereum & MPN439 & Brazil & Lücking & s. n. & $(\mathrm{F}, \mathrm{SP})$ & KM453858 & KM453795 \\
\hline P. catapastum & MPN358 & Venezuela & Lücking & 26052 & $(\mathrm{~F}, \mathrm{VEN})$ & KM453859 & JN887402 \\
\hline P. subvirescens & MPN702 & Brazil & Cáceres & 11110 & $(\mathrm{~F}, \mathrm{ISE})$ & - & KX215681 \\
\hline Pseudopyrenula aff. subgregaria & MPN288 & Panama & Lücking & $27124 b$ & $(\mathrm{~F}, \mathrm{PMA})$ & - & $\mathrm{KX} 215682$ \\
\hline P. diluta & MPN362 & Venezuela & Lücking & 26062 & $(\mathrm{~F}, \mathrm{VEN})$ & KM453861 & KM453797 \\
\hline P. diluta & MPN697 & Brazil & Lücking & 31068 & $(\mathrm{~F}, \mathrm{SP})$ & KM453862 & KM453798 \\
\hline P. endoxanthoides & MPN106 & Thailand & Lücking & 24079 & (F, RAMK) & GU327699 & GU327724 \\
\hline P. endoxanthoides & MPN573 & Thailand & Nelsen & s. n. & (F, RAMK) & KX215595 & - \\
\hline P. subgregaria & DNA1502 & Costa Rica & Lücking & 17619 & $\mathrm{CR}, \mathrm{F}, \mathrm{INB} \rightarrow \mathrm{CR})$ & DQ328997 & - \\
\hline P. subgregaria & MPN292 & Panama & Lücking & 27053 & $(\mathrm{~F}, \mathrm{PMA})$ & KM453864 & KM453800 \\
\hline P. subgregaria & MPN297 & Panama & Lücking & 27014 & $(\mathrm{~F}, \mathrm{PMA})$ & - & KX215683 \\
\hline P. subgregaria & MPN391 & USA & Nelsen & $4082 b$ & $(\mathrm{~F})$ & KM453863 & KM453799 \\
\hline P. subgregaria & MPN565 & Thailand & Nelsen & s. n. & (F, RAMK) & KX215596 & - \\
\hline P. subgregaria & MPN568 & Thailand & Nelsen & s. n. & $(\mathrm{F}, \mathrm{RAMK})$ & KX215597 & KX215684 \\
\hline P. subnudata & MPN293 & Panama & Lücking & $27014 \mathrm{r} 1$ & $(\mathrm{~F}, \mathrm{PMA})$ & KM453865 & KM453801 \\
\hline Trypethelium eluteriae & MPN111 & India & Lumbsch & $19701 \mathrm{a}$ & (F) & KM453874 & GU327726 \\
\hline T. eluteriae & MPN562 & Thailand & Nelsen & s. n. & $(\mathrm{F}, \mathrm{RAMK})$ & KX215598 & KX215685 \\
\hline T. eluteriae & MPN563 & Thailand & Nelsen & s. n. & $(\mathrm{F}, \mathrm{RAMK})$ & KX215599 & KX215686 \\
\hline T. eluteriae & MPN576 & Thailand & Nelsen & s. n. & (F, RAMK) & KX215600 & - \\
\hline T. eluteriae & MPN580 & Thailand & Parnmen & 32 & (F) & KX215601 & KX215687 \\
\hline T. foveolatum & MPN351 & Argentina & Lücking & 30515 & (CTES, F) & KM453881 & KM453816 \\
\hline T. inamoenum & MPN228 & Thailand & Lücking & 24125 & $(\mathrm{~F}, \mathrm{RAMK})$ & KM453875 & KM453810 \\
\hline T. platyleucostomum & MPN349 & Argentina & Lücking & 30512 & $(\mathrm{CTES}, \mathrm{F})$ & KM453870 & KM453806 \\
\hline T. platyleucostomum & MPN350 & Argentina & Lücking & 30512 & (CTES, F) & KX215602 & KX215688 \\
\hline T. rubroplatystomum & MPN54 & Peru & Nelsen & s. n. & $(\mathrm{F})$ & KM453871 & KM453807 \\
\hline T. rubroplatystomum & MPN64 & Peru & Nelsen & s. n. & (F) & - & KX215689 \\
\hline T. rubroplatystomum & MPN65C & Peru & Nelsen & s. n. & (F) & KX215603 & KX215690 \\
\hline T. sprengelii & MPN200B & El Salvador & Lücking & 28013 & (F, ITIC) & - & KX215691 \\
\hline T. sprengelii & MPN382 & USA & Nelsen & 4169 & (F) & KM453867 & KM453803 \\
\hline
\end{tabular}




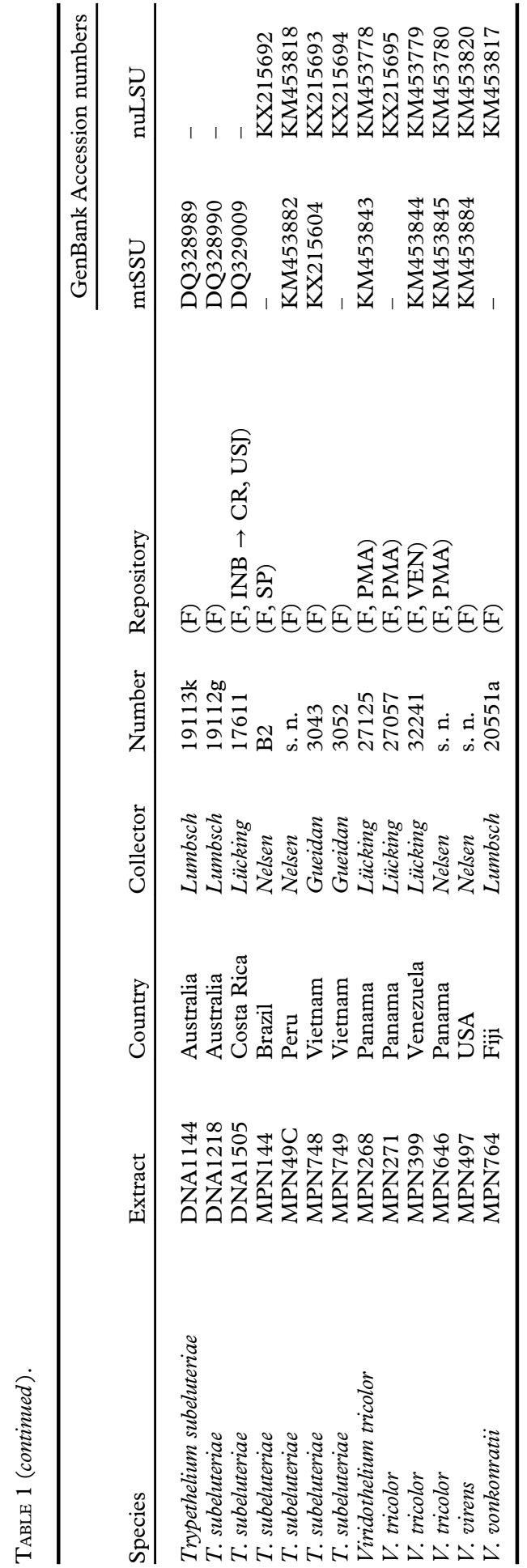

\section{Results and Discussion}

The final dataset consisted of 196 ingroup OTUs and 1272 characters (mtSSU: 690; nuLSU: 491; mtSSU PICS-Ord codes: 91). The basal portion of the best-scoring maximum likelihood tree (Figs 1 \& 2) included several clades representing the artificial genera Arthopyrenia, Fulella, and Mycomicrothelia, all with ecorticate thalli and more or less euseptate ascospores. Species formerly placed in Arthopyrenia s. lat. and Mycomicrothelia s. lat. did not form monophyletic clades and the backbone in this part of the tree was poorly supported. Therefore, while the name Bogoriella was separately reinstated for the bulk of tropical, lichenized Mycomicrothelia species (Aptroot \& Lücking 2016), here we established new genera (see below) for the wellsupported core clade of tropical, lichenized Arthopyrenia species (Constrictolumina) and for Mycomicrothelia oleosa (Novomicrothelia), for which monophyly with the other three Mycomicrothelia species (Bogoriella) was rejected (SH test, $P<0.01$ ). No formal change was proposed for Fulella fallaciosa since the available data do not allow us to conclude whether this taxon is conspecific with the type of the genus. Two further lineages representing tropical, lichenized species previously assigned to Arthopyrenia were provisionally placed in Constrictolumina, since monophyly of these lineages with the latter could not be rejected (SH test, $P>0.05$ ).

The strongly supported remainder of the tree contained lineages with astrothelioid ascospores and/or corticate thalli. This large clade was rather well supported in most parts, suggesting up to 15 lineages which we mostly interpret as distinct genera (Figs 1-4). Pseudopyrenula formed a supported, monophyletic sister group to all remaining genera which were split into two larger clades (Figs $1 \& 2$ ). The first of these two clades included Polymeridium s. str., the Trypethelium tropicum clade (Nigrovothelium), the Polymeridium proponens clade (Dictyomeridium), for which monophyly with Polymeridium s. str. was rejected ( $\mathrm{SH}$ test, $P<0.01)$, the Trypethelium virens clade (Viridothelium), Marcelaria, and Trypethelium s. str. (Fig. 2). The Trypethelium virens clade 


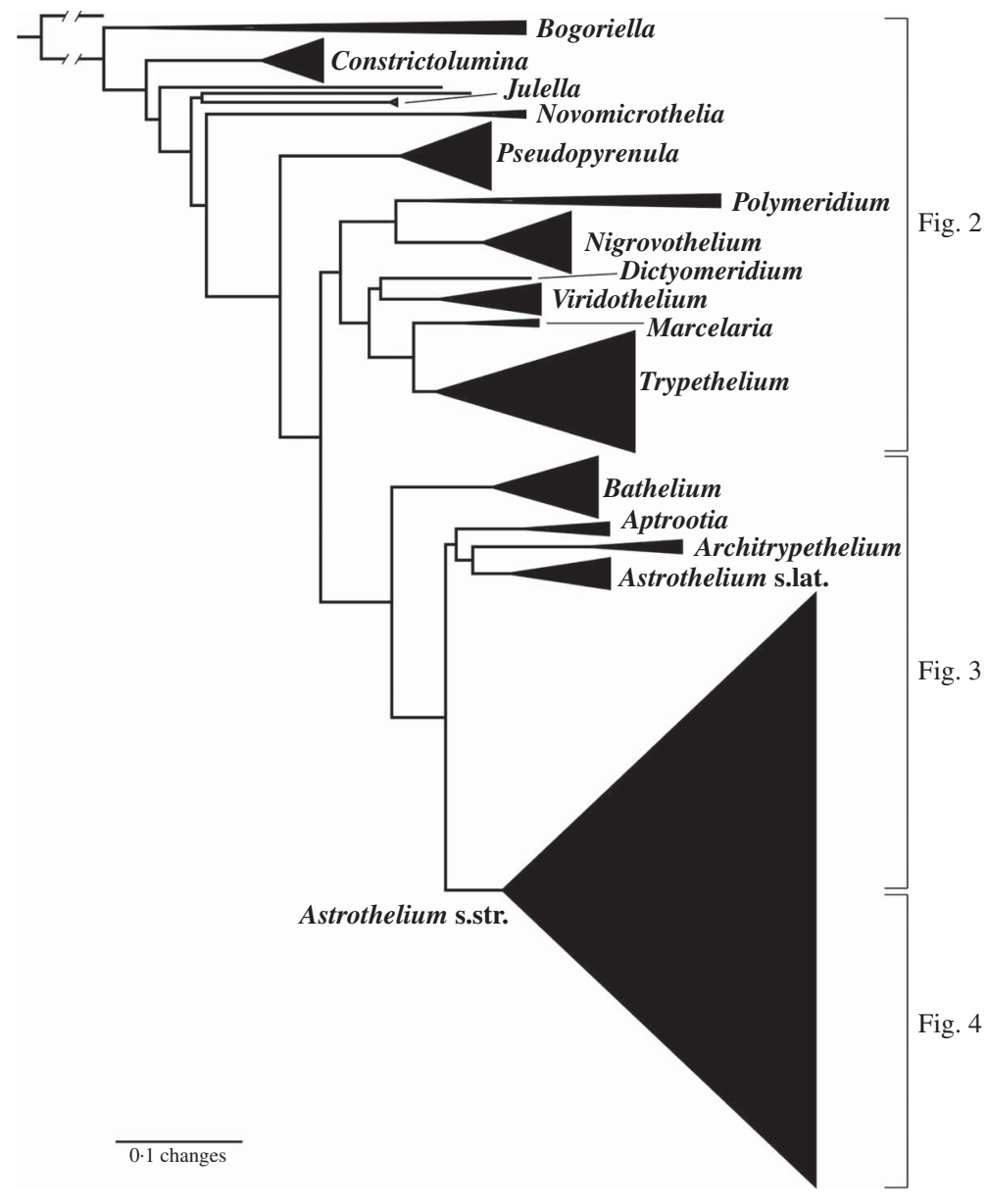

FIG. 1. Combined mtSSU-nuLSU-PICS-Ord cartoon tree of Trypetheliaceae based on maximum likelihood analysis. Accepted genera are labelled. Separate detailed figures (which also include bootstrap support values) are indicated.

had previously been identified with the name Campylothelium (Nelsen et al. 2014), but this was based on a misidentification of one of the species contained in this clade as Campylothelium puiggarii which turned out to represent an undescribed species unrelated to $C$. puiggarii; we now assume that Campylothelium falls into the Astrothelium clade although the type, Campylothelium puiggarii, has not yet been sequenced. The sister group relationship of Polymeridium s. str. with the Trypethelium tropicum clade was supported, as well as the sister group relationship of Marcelaria and Trypethelium s. str. and the position of the Polymeridium proponens (Dictyomeridium) and the Trypethelium virens (Viridothelium) clades close to Marcelaria and Trypethelium (Fig. 2).

The second clade included the genera Bathelium s. str., Aptrootia and Architrypethelium as well as a small and a large clade comprising species traditionally classified in the genera Astrothelium, Bathelium, Cryptothelium, Laurera, and Trypethelium, with Astrothelium as the oldest available name (Figs 1, $3 \& 4$ ). Bathelium s. str. formed a strongly supported sister group to the remaining clades in this portion of the tree (Fig. 3), but the relationships between 


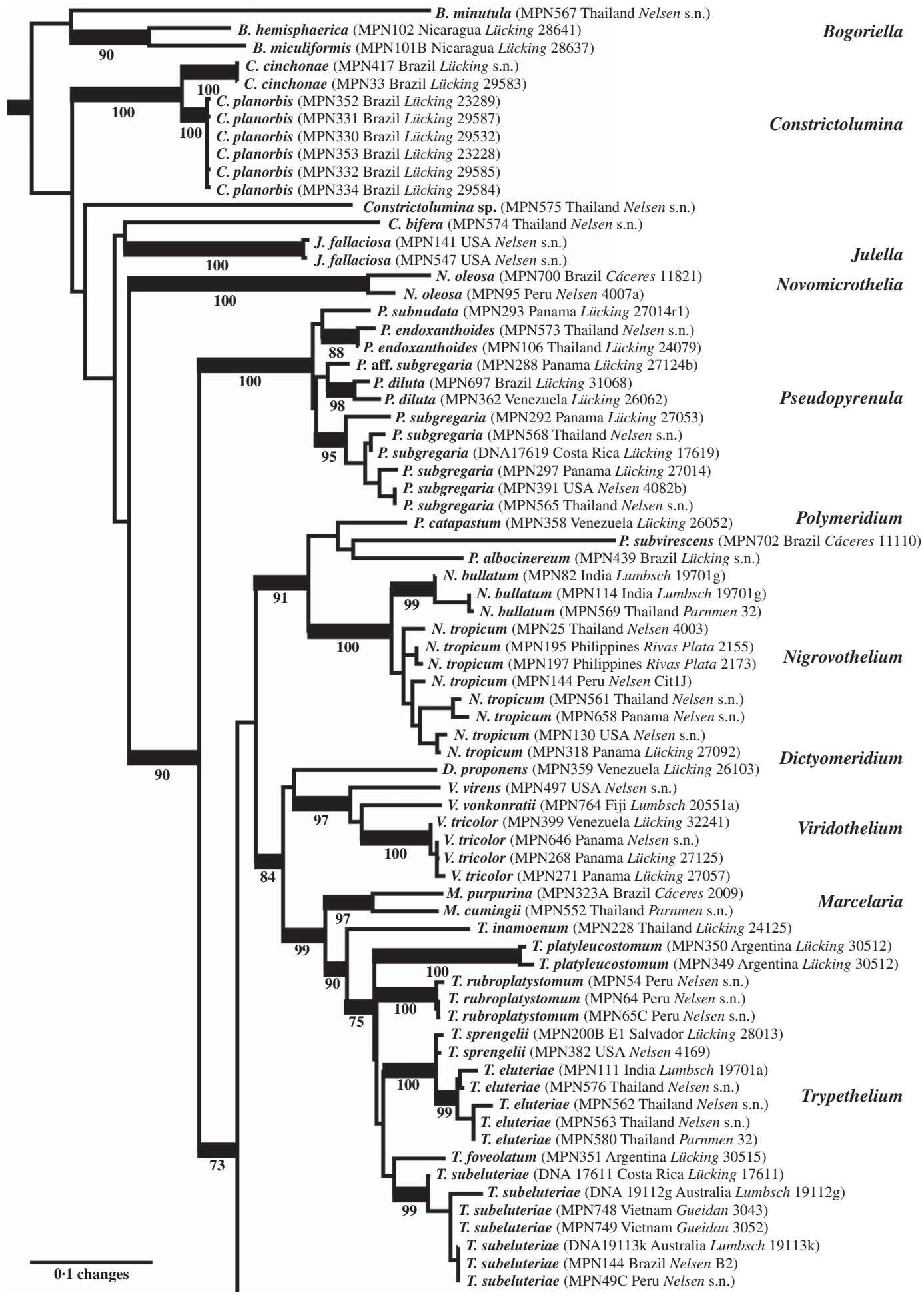


the genera Aptrootia, Architrypethelium, and Astrothelium were not supported and not fully resolved, due to the presence of a small clade of species that would morphologically be referable to Astrothelium but clustered, without support, with Aptrootia and Architrypethelium (Fig. 3). The separate mtSSU and nuLSU trees had similar topologies, with a small Astrothelium clade separated from the large Astrothelium clade; however, in the mtSSU tree, Architrypethelium was nested within the small clade and Aptrootia within the large clade (see Supplementary Materials B \& C, available online); for both loci, the alternative hypothesis that the two Astrothelium clades form a monophyletic group could not be rejected (SH test, $P>0.05$ ). Therefore, we adopted a conservative solution, retaining Aptrootia and Architrypethelium as separate genera, due to their distinctive features, and treating all other species in this large, terminal clade in a single genus, Astrothelium.

All genus-level lineages as delimited here are characterized by a combination of phenotypic features and we formally recognize 15 genera at this time within Trypetheliaceae (Aptroot \& Lücking 2016), excluding fulella (since the phylogenetic position of the type species is unknown) but including Distothelia, which has not yet been sequenced. The lineages at the base of the tree are for the time being recognized in three genera, all with ecorticate thalli, exposed, black ascomata and ascospores of variable types but not astrothelioid (i.e. lacking the endospore causing the diamond-shaped lumina characteristic of most other genera). These are Bogoriella (reinstated for tropical, lichenized species previously placed in Mycomicrothelia; ascospores euseptate, brown), Constrictolumina (for tropical, lichenized species previously placed in Arthopyrenia; ascospores subdistoseptate, often with incomplete septal invaginations, hyaline), and Novomicrothelia (for an additional species previously placed in Mycomicrothelia).
The genus fulella, previously assigned to Arthopyreniaceae or Thelenellaceae (Mayrhofer 1987; Harris 1995), has recently been placed in Halojulellaceae and Didymosphaeriaceae, respectively (Hyde et al. 2013; Ariyawansa et al. 2014) but no molecular data are yet available for the non-lichenized, European type species, f. buxi Fabre. The sequenced material falling within Trypetheliaceae fits the North American temperate species $\mathcal{F}$. fallaciosa, which might or might not be congeneric with $\mathcal{F}$. buxi, so further data are required to resolve this issue; for the time being, we do not formally accept fulella as a genus within Trypetheliaceae. The genus as currently circumscribed might well turn out to be polyphyletic, similar to Arthopyrenia s. lat. and Mycomicrothelia s. lat. A potentially available genus name for the lineage falling within Trypetheliaceae, should $\mathcal{F}$. fallaciosa prove to be unrelated to $\mathcal{F}$. buxi, is Polyblastiopsis Zahlbr. based on P. sericea (A. Massal.) Zahlbr., which appears to be related to, or conspecific with, F. fallaciosa (Purvis et al. 1992; Aptroot \& van den Boom 1995; Harris 1995).

Pseudopyrenula is characterized by a morphology similar to Bogoriella and Constrictolumina, but with astrothelioid ascospores with diamond-shaped lumina. In both morphology and position, Pseudopyrenula provides a true transitional genus between the base of the tree and the more derived taxa, combining a primitive morphology with a derived ascospore type.

The genera Polymeridium and Dictyomeridium share the plesiomorphic morphology of ecorticate thalli and largely exposed, black ascomata with the aforementioned genera and their ascospores are also euseptate. Given their supported, nested position within a clade largely characterized by astrothelioid ascospores, the euseptate ascospores of Polymeridium and Dictyomeridium could be a secondary reversal (loss of endospore) due to the ecology of these species occurring mostly in dry forest biomes (Harris 1984,

FIG. 2. Combined mtSSU-nuLSU-PICS-Ord tree of Trypetheliaceae (basal and central portion of the tree: Bogoriella to Trypethelium) based on maximum likelihood analysis. Thick lines indicate bootstrap support $\geq 70 \%$ and exact bootstrap support values are given below branches. Accepted genera are in larger font and in bold. 


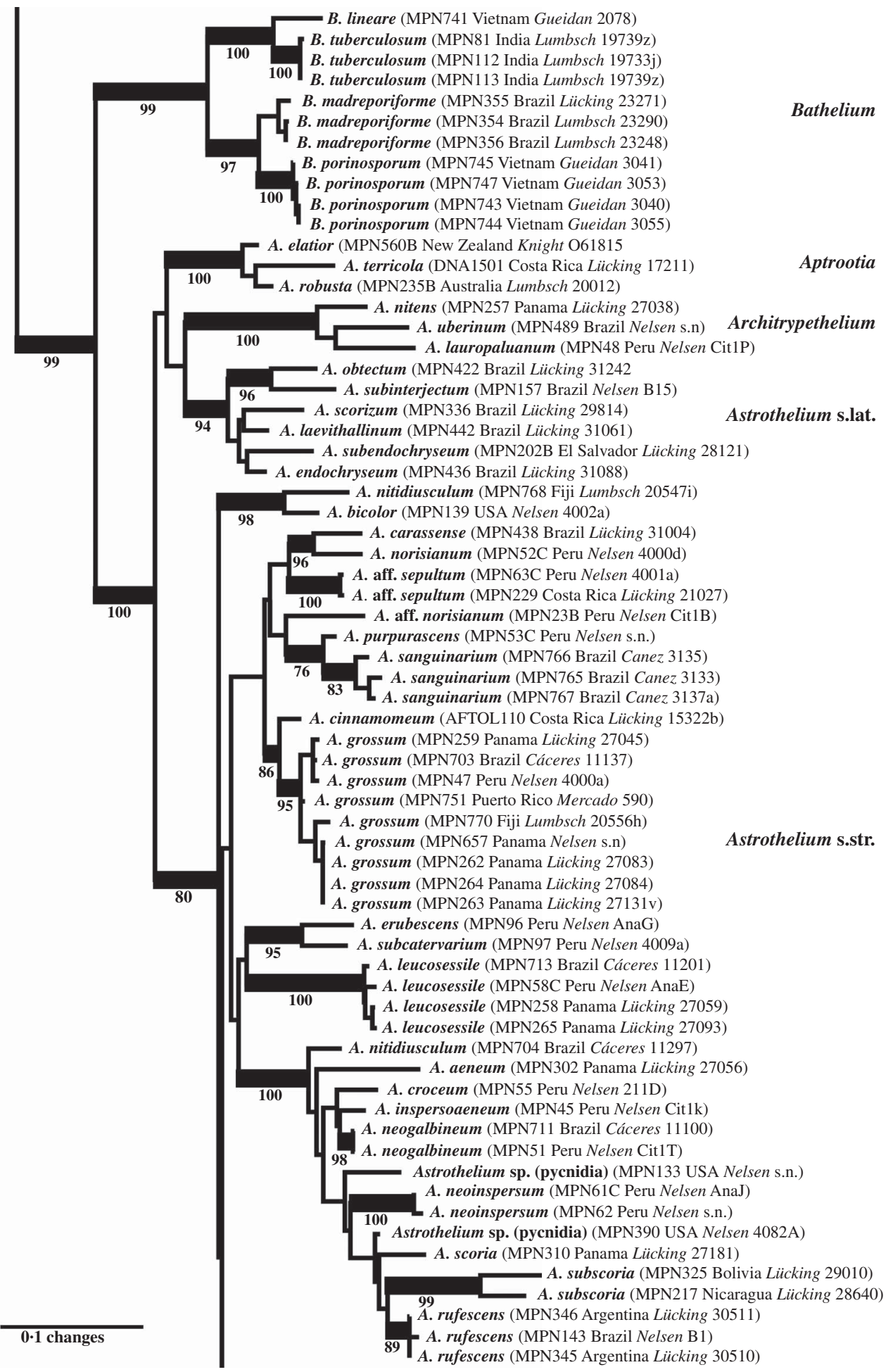


1991, 1995; Cáceres 2007; Aptroot et al. $2008,2013)$. However, this needs to be tested with a further expanded dataset, although the thin-walled ascospores of Trypethelium s. str. (see below) would support this notion. Polymeridium was initially described as a section of Arthopyrenia (Müller 1883), while Harris (1975) eventually raised the section to a separate genus within Trypetheliaceae (Tucker \& Harris 1980). The morphological distinction between Polymeridium s. str. and Dictyomeridium is not yet fully understood, but the latter appears to include species with lateral ostioles and muriform ascospores. The separate placement of this group was anticipated in earlier works (Tucker \& Harris 1980; Harris 1991), and Polymeridium proponens had at some point been assigned to Campylothelium (Tucker \& Harris 1980).

The pantropical Trypethelium tropicum complex is supported sister to Polymeridium s. str. and could be included in the latter based on topology alone, but since it differs in the corticate thallus and astrothelioid ascospores, we prefer to recognize it as a new genus, Nigrovothelium. Nigrovothelium is morphologically distinguished from Astrothelium by the completely exposed, sessile ascomata (at least partly covered by thallus or pseudostromatic in Astrothelium) and from Bathelium by the absence of pseudostromata and the black colour of the ascomata. Marcelaria has been characterized already in a separate paper (Aptroot et al. 2013) as comprising species with exposed, strongly pigmented pseudostromata, which are somewhat similar to those of Trypethelium s. str. but are not distinctly pseudostromatic and produce muriform ascospores.

Trypethelium s. str. is well delimited by its prominent to sessile pseudostromata with apical ostioles and the subdistoseptate, transversely-septate ascospores with more or less rectangular lumina, different to the astrothelioid ascospores in Astrothelium and other genera. The aforementioned notion that the euseptate ascospores in Polymeridium and Dictyomeridium could represent a secondary reversal from astrothelioid forms is in part supported by ascospore ontogeny in Trypethelium s. str., where the finally subdistoseptate ascospores pass through an astrothelioid stage early in their ontogeny (Sweetwood et al. 2012). Aptroot et al. (2008) suggested that the T. eluteriae group might be closely related to Bathelium, a hypothesis that is not supported here. Makhija \& Patwardhan (1992, 1993) suggested that Trypethelium s. str. includes subgroups with slightly different pseudostromatal anatomy: those of $T$. eluteriae and $T$. sphaerocephalum are separated from the thallus by the absence of cortical, algal and medullary layers, and instead a cortical layer is produced beneath the pseudostroma, and ascomata are surrounded by a single layer, either hyaline or filled with yellow to orange crystals. In contrast, pseudostromata in T. subeluteriae contain a cortical layer both beneath and above them.

The only conceivable morphological difference between Viridothelium and Astrothelium are the subdistoseptate versus astrothelioid ascospores. The ascospores of Viridothelium closely resemble those of Trypethelium s. str., but the two genera differ in ascoma morphology: solitary to diffusely pseudostromatic in Viridothelium and distinctly pseudostromatic in Trypethelium s. str. Possible inclusion of Viridothelium within Trypethelium s. str. is rejected by the topology (SH test, $P<0.01$ ).

The genus Bathelium is partially confirmed here as a monophyletic entity, but several species placed in this genus by Harris (1995) fall into Astrothelium, notably those with small, 3-septate ascospores and with rather low pseudostromata, such as $A$. degenerans. Bathelium s. str. instead produces strongly prominent to sessile, very conspicuous pseudostromata. This concept coincides in part with that of Trevisan (1853) and

FIG. 3. Combined mtSSU-nuLSU-PICS-Ord tree of Trypetheliaceae (central portion of the tree: Bathelium to Astrothelium p.p.) based on maximum likelihood analysis. Thick lines indicate bootstrap support $\geq 70 \%$ and exact bootstrap support values are given below branches. Accepted genera are in larger font and in bold. 


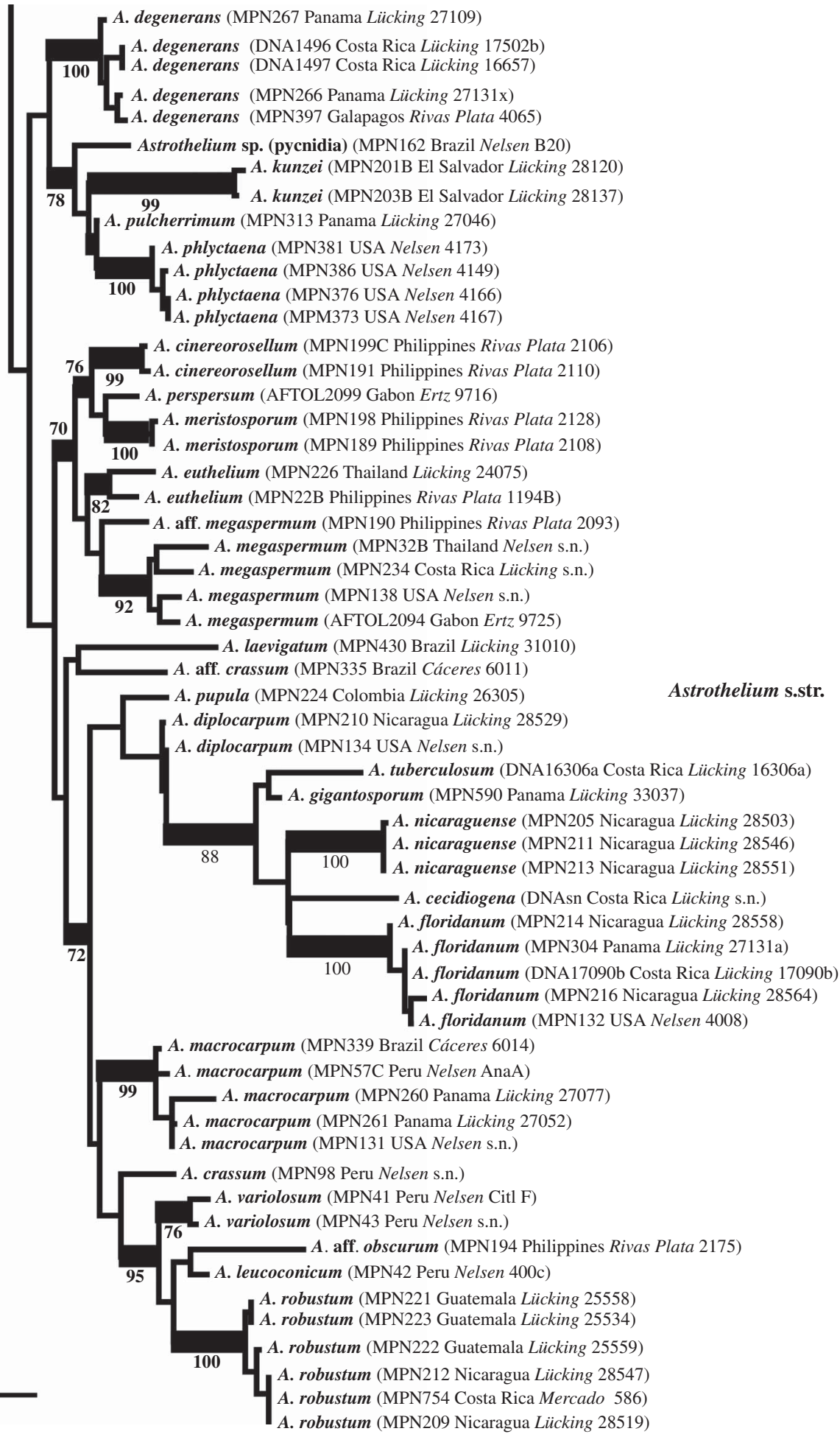


Massalongo (1860). An additional difference is that the ascospores are subdistoseptate in Bathelium and astrothelioid in Astrothelium, for example $A$. degenerans, a feature best observed in species with transversely septate ascospores.

The two genera Aptrootia and Architrypethelium, originally defined based on their peculiar ascospores (dark brown with a hard outer shell in Aptrootia and large, 3-septate and often dark brown in Architrypethelium), are confirmed as monophyletic and retained here. The ascospores of both genera pass through an astrothelioid stage before producing their characteristic mature structure (Sweetwood et al. 2012) which supports the close relationship between these genera and Astrothelium.

Finally, Astrothelium as defined here includes the bulk of species in the family, characterized by rather thick, corticate thalli and astrothelioid ascospores. The genera Campylothelium, Cryptothelium, and Laurera are now considered synonyms of this genus and most species previously classified as Trypethelium are also placed here (Aptroot $\&$ Lücking 2016). Harris (1995) had already argued that the delimitation of these genera was artificial, and predicted that many species from these genera would eventually be placed in a single genus, although he suggested Laurera (Reichenbach 1841) as a potential name, which is, however, younger than Astrothelium (Eschweiler 1824). While most of the species in this considerably emended genus have rather thick, corticate, often olive-green thalli with the ascomata usually immersed or covered by a thallus layer, much variation is found in ascoma disposition, being either solitary or aggregate or fused with either apical or lateral ostioles; also, the ascospores range from transversely septate to muriform but are without exception hyaline. While muriform ascospores usually do not display diamond-shaped lumina, their ontogeny passes through an astrothelioid stage (Sweetwood et al. 2012), explaining the observed close relationship between species with differently septate ascospores, for instance the well-known $A$. megaspermum with large, muriform ascospores, falling in a small, supported clade together with taxa with small, transversely septate ascospores, A. cinereorosellum and $A$. perspersum (Fig. 4).

The results presented here also provide a refined understanding of species delimitation in Trypetheliaceae. Characters such as thallus and ascoma morphology, hymenium inspersion, and the presence of lichexanthone, as used in Harris (1984), have been successively neglected when delimiting species, so that more recently, species were almost exclusively defined by pigments and ascospore septation and size (e.g. Harris 1991, 1995); even ascospore size was at some point considered of limited value, such as in Pseudopyrenula (Harris 1998). On the other hand, taxa such as Trypethelium eluteriae and $T$. subeluteriae have been distinguished by successive workers based on very subtle morphological and chemical differences (Makhija \& Patwardhan 1992; Harris 1995; Aptroot et al. 2008). Without independent evidence, arguments for either splitting or lumping might be valid yet remain subjective, but fortunately can be tested using a phylogenetic framework. As an example, the separation of Trypethelium eluteriae and $T$. subeluteriae was supported by our results (Fig. 2).

More importantly, we repeatedly found that species originally identified with names using available keys were located in different places in the tree, especially in the newly defined genus Astrothelium, with the commonly applied names A. (Trypethelium) aeneum, $A$. nitidiusculum, and $A$. ochroleucum (Figs $3 \& 4$ ). These morphotaxa apparently comprise many, often unrelated species, such as $A$. leucosessile, A. kunzei, and $A$. inspersaeneum (Aptroot \& Lücking 2016). Similar results were found in Pseudopyrenula and Nigrovothelium (Fig. 2), including the reinstated Pseudopyrenula endoxanthoides and the newly recognized

FIG. 4. Combined mtSSU-nuLSU-PICS-Ord tree of Trypetheliaceae (distal portion of the tree: Astrothelium p.p.) based on maximum likelihood analysis. Thick lines indicate bootstrap support $\geq 70 \%$ and exact bootstrap support values are given below branches. Accepted genera are in larger font and in bold. 
Nigrovothelium bullatum (Aptroot \& Lücking 2016; Lücking et al. 2016). In these cases, examination of the sequenced material revealed that clades were distinguished by characters such as hymenium inspersion and presence of lichexanthone, but especially by the morphology of thallus and ascomata. Thus, features such as a bullate thallus or the degree of dispersion and aggregation or emergence of the ascomata and pseudostromata appear to be species-specific. This suggests that a much more refined species concept has to be applied within the family, similar to the situation found in Graphidaceae, especially the megadiverse genera Graphis and Ocellularia, where gross morphology had been similarly neglected but was found to be diagnostic (Lücking 2009, 2014, 2015; Lücking et al. 2009). Based on these findings, the refined species concept laid out in the monographic synopsis of the family (Aptroot \& Lücking 2016) increases the number of recognized species in Trypetheliaceae based on names reinstated from prior synonymy by $c$. 70 , in addition to well over 100 new species (Aptroot \& Cáceres 2016; Aptroot et al. 2016a, b; Flakus et al. 2016; Lücking et al. 2016).

\section{Taxonomic Novelties}

\section{Constrictolumina Lücking, M. P. Nelsen \& Aptroot gen. nov.}

\section{MycoBank No.: MB 816872}

Differing from Arthopyrenia s. str. in the lichenized thallus and the ascospores with thicker walls, and usually forming secondary endospore thickenings resembling incomplete septa.

Type: Constrictolumina cinchonae (Ach.) Lücking, M. P. Nelsen \& Aptroot (holotype).

\section{Thallus not corticate.}

Ascomata single, roughly conical, erumpent to prominent and more or less exposed, not in distinct pseudostromata but sometimes fused sideways. Ostiole apical. Hamathecium hyaline, clear, filaments thick at the base, thinner above, not anastomosing. Asci clavate. Ascospores 1-3-septate, rarely submuriform, with irregular endospore formation, sometimes with pseudosepta, often one or two cells with secondary endospore invaginations resembling incomplete septa, smooth or ornamented, hyaline, very rarely becoming brownish, often becoming granular ornamented.

Pycnidia sometimes present.

Notes. This aggregate of tropical, lichenized species is separated here for the first time into a formally described genus, following the realization that this group forms part of the family Trypetheliaceae, unrelated to the non-lichenized representatives of Arthopyrenia s. str. (Nelsen et al. 2009, 2011; Hyde et al. 2013). Details of the species were given primarily by Harris (1975, 1995), as part of a variously defined genus Arthopyrenia s. lat. Including the two species sequenced and recombined here, the new genus unites nine tropical taxa treated elsewhere in this issue (Aptroot \& Lücking 2016). Constrictolumina exhibits a unique hamathecium structure different from the remainder of the Trypetheliaceae, one of the reasons why this group was not previously suspected to be related to the latter.

\section{Constrictolumina cinchonae (Ach.) Lücking, M. P. Nelsen \& Aptroot comb. nov.}

MycoBank No.: MB 816878

Verrucaria cinchonae Ach., Synops. Lich.: 90 (1814).Arthopyrenia cinchonae (Ach.) Müll. Arg., Flora 66: 287 (1883); type: "cort. Cinchonae officinalis" (H-ACH $781 \mathrm{~B}$ ! - holotype).

\section{Constrictolumina planorbis (Ach.) \\ Lücking, M. P. Nelsen \& \\ Aptroot comb. nov.}

MycoBank No.: MB 816879

Verrucaria planorbis Ach., Synops. Lich.: 92 (1814).Arthopyrenia planorbis (Ach.) Müll. Arg., Mem. Soc. Phys. Genève 30: 27 (1888); type: "cort. Crotonis Cascarillae" (H-ACH, holotype, not seen).

\section{Dictyomeridium Aptroot, M. P. Nelsen \& Lücking gen. nov.}

MycoBank No.: MB 816873 
Differing from Polymeridium s. str. in the ascomata with laterial ostiole in combination with muriform ascospores.

Type: Dictyomeridium proponens (Nyl.) Aptroot, M. P. Nelsen \& Lücking (holotype).

Thallus not corticate.

Ascomata single or a few aggregate, roughly conical to pyriform, erumpent to prominent and more or less exposed, not in distinct pseudostromata. Ostioles eccentric. Hamathecium hyaline, not inspersed. Asci with 2-8 ascospores. Ascospores muriform, hyaline, smooth, often IKI+ violet.

Pycnidia sometimes present.

Notes. This species aggregate is united here as a segregate of Polymeridium, with seven muriform-spored species with lateral ostiole (Aptroot \& Lücking 2016). While Dictyomeridium is phylogenetically separate from Polymeridium, the morphological differences are subtle, since the latter includes some species with muriform ascospores or lateral ostioles, but not in combination. The type of Dictyomeridium, D. proponens, representing the most common species of the genus, was for a long time recognized under different names in the genus Campylothelium.

\section{Dictyomeridium proponens (Nyl.) Aptroot, M. P. Nelsen \& Lücking comb. nov.}

MycoBank No.: MB 816880

Verrucaria proponens Nyl., Bull. Soc. Linn. Normandie, sér. 2 2: 130 (1868).- Polyblastia proponens (Nyl.) Müll. Arg. Flora 65: 402 (1882).-Campylothelium proponens (Nyl.) Müll. Arg., Hedwigia 31: 286 (1892)._Polyblastiopsis proponens (Nyl.) Zahlbr., Catal. Lich. Univ. 1: 351 (1922).-Polymeridium proponens (Nyl.) R. C. Harris, Bol. Mus. Paraense Emilio Goeldi, Ser. Bot., 7: 637 ('1991') [1993]; type: New Caledonia, Lifu, Loyalty Islands, Thiebaut. (H-NYL-holotype, not seen).

\section{Nigrovothelium Lücking, M. P. Nelsen \& Aptroot gen. nov.}

\section{MycoBank No.: MB 816875}

Differing from Polymeridium in the corticate thallus and astrothelioid ascospores, from Bathelium in the mostly single, black ascomata and astrothelioid ascospores, and from Astrothelium in the fully exposed, sessile, black ascomata.

Type: Nigrovothelium tropicum (Ach.) Lücking, M. P. Nelsen \& Aptroot (holotype).

\section{Thallus corticate.}

Ascomata usually single but often crowded and irregularly confluent, sessile, ovoid, not in pseudostromata. Ostiole apical. Hamathecium hyaline, clear, filaments thin, anastomosing paraphysoids, often inspersed with oil. Asci clavate. Ascospores transversely 3-septate, with distinct endospore formation creating diamondshaped lumina (astrothelioid), hyaline.

Notes. The genus is described here to accommodate Trypethelium tropicum and at least one additional, newly recognized species (Lücking et al. 2016). While this genus resembles other genera in certain characters, such as Pseudopyrenula and Astrothelium in the astrothelioid ascospores and Bathelium in the exposed, dark ascomata, its unique combination of characters and its phylogenetic position, sister to the morphologically distinct Polymeridium, merit its recognition as a separate taxon.

\section{Nigrovothelium tropicum (Ach.) \\ Lücking, M. P. Nelsen \& Aptroot comb. nov.}

MycoBank No.: MB 816881

Verrucaria tropica Ach., Lichenogr. Univ.: 278 (1810).Sagedia tropica (Ach.) A. Massal., Ricerch. Auton. Lich.: 161 (1852).- Pyrenula tropica (Ach.) Trevis., Spighe e Paglie: 17 (1853).- Spermatodium tropicum (Ach.) Trevis., Conspect. Verruc.: 11 (1860).-Pseudopyrenula tropica (Ach.) Müll. Arg., Flora 66: 248 (1883).-Trypethelium tropicum (Ach.) Müll. Arg., Bot. Fahrb. Syst. 6: 393 (1885); type: America, Swartz (H-ACH 707A!-lectotype, designated here; $\mathrm{BM}-\mathrm{ACH}$-isolectotype).

\section{Novomicrothelia Aptroot, M. P. Nelsen \& Lücking gen. nov.}

MycoBank No.: MB 816876

Similar to Bogoriella but forming a separate phylogenetic clade, with the following substitutions in the large subunit nuclear ribosomal DNA (nuLSU; relative positions following Supplementary Material A, available online): 46, 47, 308, 449, 458 (A replaces C); 117, 298, 379, 397, 447, 459 (A replaces G); 32, 68, 81, 109-111, 311, 316, 355 
(A replaces $\mathrm{T}) ; 45,70(\mathrm{C}$ replaces $\mathrm{A}) ; 98$ (C replaces $\mathrm{G})$; 41, 87, 106, 195, 196, 351, 419, 457, 461 (C replaces T); 36, 383 (G replaces A); 322 (G replaces C); 44 (G replaces T); 83, 266, 307, 321, 361 (T replaces A); 40, 97, 320, 385, 440, 474 ( $\mathrm{T}$ replaces $\mathrm{C}$ ); 352, 354 ( $\mathrm{T}$ replaces $\mathrm{G}$ ); also differing from most species of Bogoriella in the inspersed hamathecium and ascospore wall invaginations besides the (sub-)median septum.

Type: Novomicrothelia oleosa (Aptroot) Aptroot, M. P. Nelsen \& Lücking (holotype).

Thallus not corticate.

Ascomata single, roughly conical, erumpent to prominent and more or less exposed, not in pseudostromata. Ostiole apical. Hamathecium hyaline, inspersed with oil droplets, filaments thin, anastomosing paraphysoids. Asci clavate. Ascospores transversely 1-septate, with irregular endospore formation, becoming ornamented, brown, rather elongated.

Pycnidia sometimes present.

Discussion. The genus is described here to accommodate a single species that was until now united with temperate, non-lichenized fungi in the genus Mycomicrothelia. Novomicrothelia is phylogenetically distinct from the morphologically similar, reinstated genus Bogoriella but the phenotypic distinction between the two genera is not very clear yet; more data are needed for these basal lineages within the family to fully understand their phylogeny and classification. According to Harris (1995), N. oleosa is unique based on its inspersed hamathecium and ascospore wall invaginations similar to those found in Constrictolumina but the small number of species of Bogoriella sequenced so far does not allow us to conclude whether these are consistent differences. Two species currently accepted in Bogoriella have an inspersed hamathecium (Aptroot \& Lücking 2016) but these have not yet been sequenced.

Since the phenotypic differences between Novomicrothelia and Bogoriella are not yet clear, but both form distantly related clades for which monophyly as a single clade was rejected, we provided diagnostic molecular features as allowed by the Code. The Code specified in such a case that the differential characters (i.e. relative columns and substitutions) need to be spelled out, and we provide a possible model for this case. It is obvious that further data might change these characters, in particular reduce the number of diagnostic columns, but since this applies analogously to any phenotypic characters when further data are added, it does not make the diagnosis invalid.

\section{Novomicrothelia oleosa (Aptroot) \\ Aptroot, M. P. Nelsen \& Lücking comb. nov.}

MycoBank No.: MB 816882

Mycomicrothelia oleosa Aptroot, Biblioth. Lichenol. 44: 133 (1991); type: Trinidad, Caroni, north bank road, Britton et al. 869 (NY!-holotype).

\section{Viridothelium Lücking, M. P. Nelsen \& Aptroot gen. nov.}

MycoBank No.: MB 816877

Differing from Astrothelium in the subdistoseptate ascospores and from Trypethelium s. str. in the absence of well-defined pseudostromata.

Type: Viridothelium virens (Tuck. ex Michener) Lücking, M. P. Nelsen \& Aptroot (holotype).

Thallus corticate, often warted.

Ascomata simple or aggregated in pseudostromata, which can be hardly to clearly raised and are usually not of a different structure and colour from the thallus. Ostioles apical or eccentric, simple or fused. Wall hyphal (textura intricata), usually carbonized. Hamathecium inspersed with oil droplets or not, filaments thin, anastomosing paraphysoids. Ascospores subdistoseptate, with thin walls and only slightly thickened septa, hyaline, I- or weakly I+ violet-blue, transversely septate.

\section{Pycnidia occasionally present.}

Notes. This new genus accommodates Trypethelium virens, a taxon that has long been considered unique due to its northern temperate distribution and the non-astrothelioid, I+ weakly amyloid ascospores (Aptroot \& Lücking 2016; Aptroot et al. $2016 a, b$; Lücking et al. 2016); several further, tropical species are also included in this clade. Viridothelium is superficially similar to Astrothelium but appears in a distant phylogenetic position; the main difference lies in 
the subdistoseptate ascospores resembling those of Trypethelium s. str.

\section{Viridothelium virens (Tuck. ex Michener) Lücking, M. P. Nelsen \& Aptroot comb. nov.}

\author{
MycoBank No.: MB 816883
}

Trypethelium virens Tuck. ex Michener, W. Dard. Fl. Cest. ed. 3: 453 (1853).- Trypethelium eluteriae var. virens (Tuck. ex Michener) Trevis., Flora 44: 20 (1861); type: USA, Arkansas, Dardanelle, Michener, 1853 (FH-holotype, not seen; M! - isotype).

We are grateful to a number of organizations for funding including: NSF-DEB 0715660 "Neotropical Epiphytic Microlichens - An Innovative Inventory of a Highly Diverse yet Little Known Group of Symbiotic Organisms" to The Field Museum (PI Robert Lücking), a grant from the Committee on Evolutionary Biology (University of Chicago) to $\mathrm{MN}$, and the Caterpillar ${ }^{\circledR}$ Company provided funds to study lichens from Panama. The American Society of Plant Taxonomists is also acknowledged for a Graduate Student Research Grant awarded to MN. Additionally, MN's work was supported the University of Chicago and by a Brown Family Fellowship through the Field Museum, as well as by a William Harper Rainey Fellowship through the University of Chicago. The CNPq (Conselho Nacional de Desenvolvimento Científico e Tecnológico) is thanked for a research grant and field trip funding (Processos 311706/ 2012-6 and CNPq-Sisbiota Processo 563342/2010-2) to MESC. As part of the Census of Galapagos Biodiversity, the Galapagos Lichen Inventory received funds from several donors (for a detailed list see http://www.darwinfoundation. org/datazone/checklists). The lichen inventory in particular received funds from The Bay and Paul Foundations, the Erwin Warth Stiftung, NSF (grant no. DEB 0841405) and, most recently, the Mohamed bin Zayed Species Conservation Fund, project no. 152510692. This publication is contribution number 2143 of the Charles Darwin Foundation for the Galapagos Islands. We especially thank Galo Quedaza and Victor Carrión from the Galapagos National Park for technical support and specimen export permits for Galapagos material analyzed in this study. Logistical support was provided by the University of Panama (Department of Botany) in the development of two lichen seminars $(2009,2011)$ under the program Neotropical Epiphytic Microlichens in which the lichens were collected. Thanks also to Park authorities and rangers from Parque Nacional Altos de Campana during the field trips and to the Ministry of the Environment (former A.N.A.M.) for collection and export permits. The Universidad Distrital Francisco José de Caldas in Bogotá, Colombia, is thanked for providing support to $\mathrm{BM}$ as part of the program 'Mobilidad Académica' for professors. The Natural History Museum in London is thanked for research and travel funds to CG and the Vietnam National Museum of Nature in Hanoi for organizing the fieldwork.
SuPPlementary MATERIAL

For supplementary material accompanying this paper visit http://dx.doi.org/10.1017/S0024282916000505

\section{REFERENCES}

Aptroot, A. (1991a) Tropical pyrenocarpous lichens. A phylogenetic approach. In Tropical Lichens: Their Systematics, Conservation and Ecology (D. J. Galloway, ed): 253-273. Oxford: Clarendon Press.

Aptroot, A. (1991b) A monograph of the Pyrenulaceae (excluding Anthracothecium and Pyrenula) and the Requienellaceae, with notes on the Pleomassariaceae, the Trypetheliaceae and Mycomicrothelia (lichenized and non-lichenized Ascomycetes). Bibliotheca Lichenologica 44: $1-178$.

Aptroot, A. (2009a) Trypetheliaceae. Flora of Australia 57: 535-552.

Aptroot, A. (2009b) Diversity and endemism in the pyrenocarpous lichen families Pyrenulaceae and Trypetheliaceae in the Malesian flora region. Blumea 54: $145-147$.

Aptroot, A. \& Cáceres, M. E. S. (2016) New Trypetheliaceae from the Amazon basin in Rondonia (Brazil), the centre of diversity of the genus Astrothelium. Lichenologist 48: 693-712.

Aptroot, A. \& Lücking, R. (2016) A revisionary synopsis of the Trypetheliaceae (Ascomycota: Trypetheliales). Lichenologist 48: 763-982.

Aptroot, A. \& Schumm, F. (2012) The genus Melanophloea, an example of convergent evolution towards polyspory. Lichenologist 44: 501-509.

Aptroot, A. \& van den Boom, P. P. G. (1995) Strigula lateralis sp. nov. with notes on the genus fulella (Ascomycetes). Mycotaxon 56: 1-8.

Aptroot, A., Lücking, R., Sipman, H. J. M., Umaña, L. \& Chaves, J. L. (2008) Pyrenocarpous lichens with bitunicate asci: a first assessment of the lichen biodiversity inventory of Costa Rica. Bibliotheca Lichenologica 97: 1-162.

Aptroot, A., Menezes, A. A., Lima, E. L., Xavier-Leite, A. B. \& Cáceres, M. E. S. (2013) New species of Polymeridium from Brazil expand the range of known morphological variation within the genus. Lichenologist 45: 545-552.

Aptroot, A., Ertz, D., Etayo, J. A. S., Gueidan, C., Mercado-Díaz, J. A., Schumm, F. \& Weerakoon, G. (2016a) Forty-six new species of Trypetheliaceae from the tropics. Lichenologist 48: 609-638.

Aptroot, A., Mendonça, C. O., Andrade, D. S., Silva, J. R., Martins, S. M. A., Gumboski, E., Fraga Júnior, C. A. V. \& Cáceres, M. E. S. (2016b) New Trypetheliaceae from northern and southern Atlantic rainforests in Brazil. Lichenologist 48: 713-725.

Ariyawansa, H. A., Tanaka, K., Thambugala, K. M., Phookamsak, R., Tian, Q., Camporesi, E., Hongsanan, S., Monkai, J., Wanasinghe, D. N., Mapook, A., et al. (2014) A molecular phylogenetic 
reappraisal of the Didymosphaeriaceae (= Montagnulaceae). Fungal Diversity 68: 69-104.

Barr, M. E. (1979) A classification of Loculoascomycetes. Mycologia 71: 935-957.

Barr, M. E. (1987) Prodromus to Class Loculoascomycetes. Amherst, USA: Published by the author.

Berbee, M. (1996) Loculoascomycete origins and evolution of filamentous ascomycete morphology based on 18S rRNA gene sequence data. Molecular Phylogenetics and Evolution 13: 462-470.

Cáceres, M. E. S. (2007) Corticolous crustose and microfoliose lichens of northeastern Brazil. Libri Botanici 22: 1-168.

Cannon, P. F. \& Kirk, P. M. (2007) Fungal Families of the World. Wallingford: CABI Publishing.

Culberson, W. L. \& Culberson, C. F. (1970) A phylogenetic view of chemical evolution in the lichens. Bryologist 73: 1-31.

Del Prado, R., Schmitt, I., Kautz, S., Palice, Z., Lücking, R. \& Lumbsch, H. T. (2006) Molecular data place Trypetheliaceae in Dothideomycetes. Mycological Research 110: 511-520.

Eriksson, O. E. (1981) The families of bitunicate ascomycetes. Opera Botanica 60: 1-220.

Eriksson, O. E., Barah, H.-O., Currah, R. S., Hansen, K., Kurtzman, C. P., Rambold, G. \& Laessøe, T. (2004) Outline of Ascomycota. Myconet 10: 1-99.

Ertz, D., Diederich, P., Lawrey, J. D., Berger, F., Freebury, C. E., Coppins, B., Gardiennet, A. \& Hafellner, J. (2015) Phylogenetic insights resolve Dacampiaceae (Pleosporales) as polyphyletic: Didymocyrtis (Pleosporales, Phaeosphaeriaceae) with Phoma-like anamorphs resurrected and segregated from Polycoccum (Trypetheliales, Polycoccaceae fam. nov.). Fungal Diversity 74: 53-89.

Eschweiler, F. G. (1824) Systema Lichenum: Genera Exhibens Rite Distincta, Pluribus Novis Adaucta. Nuremberg: J. L. Schrag.

Flakus, A., Kukwa, M. \& Aptroot, A. (2016) Trypetheliaceae of Bolivia: an updated checklist with descriptions of twenty-four new species. Lichenologist 48: 661-692.

Harris, R. C. (1975) A taxonomic revision of the genus Arthopyrenia Massal. s. lat. (Ascomycetes) in North America. Ph.D. thesis, Michigan State University.

Harris, R. C. (1984) The family Trypetheliaceae (Loculoascomycetes: lichenized Melanommatales) in Amazonian Brazil. Acta Amazonica 14 (Supplement): 55-80.

Harris, R. C. (1989a) Working Keys to the Lichen-Forming Fungi of Puerto Rico. Tropical lichen workshop, presented at the Catholic University of Puerto Rico. Bronx, New York: Published by the author.

Harris, R. C. (1989b) A sketch of the family Pyrenulaceae (Melanommatales) in eastern North America. Memoirs of the New York Botanical Garden 49: 74-107.

Harris, R. C. (1990) Some Florida Lichens. Bronx, New York: Published by the author.

Harris, R. C. (1991) A revision of Polymeridium (Muell. Arg.) R. C. Harris (Trypetheliaceae). Boletim do
Museu Paraense Emilio Goeldi. Série Botânica 7: 619-644.

Harris, R. C. (1995) More Florida Lichens. Including the $10 \phi$ Tour of the Pyrenolichens. Bronx, New York: Published by the author.

Harris, R. C. (1998) A preliminary revision of Pseudopyrenula Müll Arg. (lichenized ascomycetes, Trypetheliaceae) with a redisposition of the names previously assigned to the genus. In Lichenographia Thomsoniana: North American Lichenology (M. G. Glenn, R. C. Harris, R. Dirig \& M. S. Cole, eds): 133-148. Ithaca: Mycotaxon Ltd.

Hyde, K. D., Jones, E. B. G., Liu, J.-K., Ariyawansha, H., Boehm, E., Boonmee, S., Braun, U., Chomnunti, P., Crous, P. W., Dai, D., et al. (2013) Families of Dothideomycetes. Fungal Diversity 63: 1-313.

Katoh, K., Asimenos, G. \& Toh, H. (2009) Multiple alignment of DNA sequences with MAFFT. Methods in Molecular Biology 537: 39-64.

Kauff, F. \& Lutzoni, F. (2002) Phylogeny of the Gyalectales and Ostropales (Ascomycota: Fungi): among and within order relationships based on nuclear ribosomal RNA small and large subunits. Molecular Phylogenetics and Evolution 25: 138-156.

Kauff, F. \& Lutzoni, F. (2003) Compat.py - a program to detect topological conflict between supported clades in phylogenetic trees. Available at: http://www.lutzonilab. net/pages/download.shtml.

Kirk, P. M., Cannon, P. F., David, J. C. \& Stalpers, J. A. (2001) Ainsworth E Bisby's Dictionary of the Fungi, 9th Edition. Wallingford: CABI Publishing.

Komposch, H. \& Hafellner, J. (2000) Diversity and vertical distribution of lichens in a Venezuelan tropical lowland rain forest. Selbyana 21: 11-24.

Komposch, H. \& Hafellner, J. (2003) Species composition of lichen dominated corticolous communities: a lowland rain forest canopy compared to an adjacent shrubland in Venezuela. Bibliotheca Lichenologica 86: 351-367.

Komposch, H., Aptroot, A. \& Hafellner, J. (2002) New species of lichenized and non-lichenized ascomycetes from canopy in southern Venezuela. Lichenologist 34: 223-235.

Letrouit-Galinou, M.-A. (1957) Revision monographique du genre Laurera (Lichens, Trypéthéliacées). Revue Bryologique et Lichénologique 26: 207-264.

Letrouit-Galinou, M.-A. (1958) Revision monographique du genre Laurera (Lichens, Trypéthéliacées). Supplément I. Revue Bryologique et Lichénologique 27: 66-73.

Liew, E. C. Y., Aptroot, A. \& Hyde, K. D. (2000) Phylogenetic significance of the pseudoparaphyses in Loculoascomycete taxonomy. Molecular Phylogenetics and Evolution 16: 392-402.

Lücking, R. (2009) The taxonomy of the genus Graphis sensu Staiger (Ascomycota: Ostropales: Graphidaceae). Lichenologist 41: 319-362.

Lücking, R. (2014) A key to species of the Ocellularia papillata, perforata and terebrata morphodemes (Ascomycota: Graphidaceae). Glalia 6(3):1-35. 
Lücking, R. (2015) Thelotremoid Graphidaceae from the NYBG herbarium: new species, range extensions, and a forgotten lichen. Opuscula Philolichenum 14: 1-57.

Lücking, R., Sipman, H. J. M., Umaña, L., Chaves, J.-L. \& Lumbsch, H. T. (2007) Aptrootia (Dothideomycetes: Trypetheliaceae), a new genus of pyrenocarpous lichens for Thelenella terricola. Lichenologist 39: 187-193.

Lücking, R., Archer, A. W. \& Aptroot, A. (2009) A world-wide key to the genus Graphis (Ostropales: Graphidaceae). Lichenologist 41: 363-452.

Lücking, R., Hodkinson, B. P., Stamatakis, A. \& Cartwright, R. A. (2011) PICS-Ord: unlimited coding of ambiguous regions by pairwise identity and cost scores ordination. Bioinformatics 12: 10.

Lücking, R., Nelsen, M. P., Aptroot, A., Benatti, M. N., Binh, N. Q., Gueidan, C., Gutiérrez, M. C., Jungbluth, P., Lumbsch, H. T., Marcelli, M. P., et al. (2016) A pot-pourri of new species of Trypetheliaceae resulting from molecular phylogenetic studies. Lichenologist 48: 639-660.

Lumbsch, H. T. \& Huhndorf, S. M. (2010) Myconet Volume 14. Part One. Outline of Ascomycota - 2009. Fieldiana, Life and Earth Sciences 1: 1-42.

Lumbsch, H. T. \& Lindemuth, R. (2001) Major lineages of Dothideomycetes (Ascomycota) inferred from SSU and LSU rDNA sequences. Mycological Research 105: 901-908.

Lutzoni, F., Kauff, F., Cox, C. J., McLaughlin, D., Celio, G., Dentinger, B., Padamsee, M., Hibbett, D., James, T. Y., Baloch, E., et al. (2004) Assembling the fungal tree of life: progress, classification, and evolution of subcellular traits. American fournal of Botany 91: 1446-1480.

Makhija, U. \& Patwardhan, P. G. (1988) The lichen genus Laurera (family Trypetheliaceae) in India. Mycotaxon 31: 565-590.

Makhija, U. \& Patwardhan, P. G. (1992) Nomenclatural notes on some species of Trypethelium. International fournal of Mycology and Lichenology 5: 237-251.

Makhija, U. \& Patwardhan, P. G. (1993) A contribution to our knowledge of the lichen genus Trypethelium (family Trypetheliaceae). Fournal of the Hattori Botanical Laboratory 73: 183-219.

Mangold, A., Martín, M. P., Lücking, R. \& Lumbsch, H. T. (2008) Molecular phylogeny suggests synonymy of Thelotremataceae within Graphidaceae (Ascomycota: Ostropales). Taxon 57: 476-486.

Manojlovic, N. T., Vasiljevic, P. J., Gritsanapan, W., Supabphol, R. \& Manojlovic, I. (2010) Phytochemical and antioxidant studies of Laurera benguelensis growing in Thailand. Biological Research 43: $169-176$.

Massalongo, A. B. (1860) Esame comparativo di alcuni generi di licheni. Atti del Reale Istituto Veneto di Scienze, Lettere ed Arti, Ser. 3 5: 313-337.

Mathey, A. \& Hoder, D. (1978) Distribution of lichen substances by means of fluorescence microscopy, cathodoluminescence in scanning electron microscopy and X-ray microanalysis in Lecanora-, Buellia-, Laurera- and Trypethelium-species. Nova Hedwigia 30: 127-138.
Mathey, A. \& Lukins, P. B. (2001) Spatial distribution of perylenequinones in lichens and extended quinones in quincyte using confocal fluorescence microscopy. Micron 32: 107-113.

Mathey, A., Van Vaeck, L. \& Steglich, W. (1987) Investigation of semi-thin cryosections of lichens by laser microprobe mass spectrometry. Analytica Chimica Acta 195: 89-96.

Mathey, A., Van Roy, W., Van Vaeck, L., Eckhardt, G. \& Steglich, W. (1994) In situ analysis of a new perylene quinone in lichens by fourier-transform laser microprobe mass spectrometry with external source. Rapid Communications in Mass Spectrometry 8: 46-52.

Mayrhofer, H. (1987) Monographie der Flechtengattung Thelenella. Bibliotheca Lichenologica 26: 1-106.

Müller, J. (1883) Lichenologische Beiträge 18. Flora 66: 317-322.

Nelsen, M. P., Lücking, R., Grube, M., Mbatchou, J. S., Muggia, L., Rivas Plata, E. \& Lumbsch, H. T. (2009) Unravelling the phylogenetic relationships of lichenised fungi in Dothideomyceta. Studies in Mycology 64: 135-144.

Nelsen, M. P., Lücking, R., Mbatchou, J. S., Andrew, C. J., Spielmann, A. A. \& Lumbsch, H. T. (2011) New insights into relationships of lichen-forming Dothideomycetes. Fungal Diversity 51: 155-162.

Nelsen, M. P., Lücking, R., Andrew, C. J., Rivas Plata, E., Chaves, J. L., Cáceres, M. E. S. \& Ventura, N. (2012) Dismantling Herpothallon: Herpothallon antillarum (Arthoniomycetes: Arthoniaceae) is a member of the genus Diorygma (Lecanoromycetes: Graphidaceae). Bryologist 115: 313-321.

Nelsen, M. P., Lücking, R., Aptroot, A., Andrew, C. J., Cáceres, M. E. S., Rivas Plata, E., Gueidan, C., da Silva Canêz, L., Knight, A., Ludwig, L. R., et al. (2014) Elucidating phylogenetic relationships and genus-level classification within the fungal family Trypetheliaceae (Ascomycota: Dothideomycetes). Taxon 63: 974-992.

Penn, O., Privman, E., Ashkenazy, H., Landan, G., Graur, D. \& Pupko, T. (2010a) GUIDANCE: a web server for assessing alignment confidence scores. Nucleic Acids Research 38: W23-W28.

Penn, O., Privman, E., Landan, G., Graur, D. \& Pupko, T. (2010b) An alignment confidence score capturing robustness to guide tree uncertainty. Molecular Biology and Evolution 27: 1759-1767.

Purvis, O. W., Coppins, B. J., Hawksworth, D. L., James, P. W. \& Moore, D. M. (eds) (1992) The Lichen Flora of Great Britain and Ireland. London: Natural History Museum Publications \& British Lichen Society.

Reichenbach, H. G. L. (1841) Deutsche Botaniker. Erster Band. Das Herbarienbuch: Erklärung des natürlichen Pflanzensystems, systematische Aufzählung, Synonymik und Register der bis jetzt bekannten Pflanzengattungen. Dresden, Leipzig: In der Arnoldischen Buchhandlung.

Schoch, C. L., Crous, P. W., Groenewald, J. Z., Boehm, E. W. A., Burgess, T. I., de Gruyter, J., de Hoog, G. S., Dixon, L. J., Grube, M., Gueidan, C., et al. 
(2009) A class-wide phylogenetic assessment of Dothideomycetes. Studies in Mycology 64: 1-15.

Stamatakis, A. (2006) RAxML-VI-HPC: maximum likelihood-based phylogenetic analyses with thousands of taxa and mixed models. Bioinformatics 22: 2688-2690.

Stamatakis, A., Hoover, P. \& Rougemont, Y. J. (2008) A rapid bootstrap algorithm for the RAxML web servers. Systematic Biology 57: 758-771.

Stensiö, K.-E. \& Wachtmeister, C. A. (1969) 1,5,8trihydroxy-6-methoxy-3-methylantraquinone from Laurera purpurina (Nyl.) Zahlbr. Acta Chemica Scandinavica 23: 144-148.

Sweetwood, G., Lücking, R., Nelsen, M. P. \& Aptroot, A. (2012) Ascospore ontogeny and discharge in megalosporous Trypetheliaceae and Graphidaceae (Ascomycota: Dothideomycetes and Lecanoromycetes) suggest phylogenetic relationships and ecological constraints. Lichenologist 44: 277-296.

Trevisan, V. (1853) Caratteri essenziali di dodici nuovi generi di Licheni. In Spighe e Paglie, Scritti Botanici Varii. I (V. Trevisan, ed.): 7-20. Padova: Sicca.
Tucker, S. C. \& Harris, R. C. (1980) New and noteworthy pyrenocarpous lichens from Louisiana and Florida. Bryologist 83: 1-20.

Vilgalys, R. \& Hester, M. (1990) Rapid genetic identification and mapping of enzymatically amplified ribosomal DNA from several Cryptococcus species. Fournal of Bacteriology 172: 4238-4246.

Weerakoon, G., Aptroot, A., Lumbsch, H. T., Wolseley, P. A., Wijeyaratne, S. C. \& Gueidan, C. (2012) New molecular data on Pyrenulaceae from Sri Lanka reveal two well-supported groups within this family. Lichenologist 44: 639-647.

Zhou, S. \& Stanosz, G. R. (2001) Primers for the amplification of $\mathrm{mt}$ SSU rDNA, and a phylogenetic study of Botryosphaeria and associated anamorphic fungi. Mycological Research 105: 1033-1044.

Zoller, S., Scheidegger, C. \& Sperisen, C. (1999) PCR primers for the amplification of mitochondrial small subunit ribosomal DNA of lichen-forming ascomycetes. Lichenologist 31: 511-516. 